\title{
Human stem cell-based models for studying autism spectrum disorder-related neuronal dysfunction
}

\author{
Arquimedes Cheffer ${ }^{1 \dagger}$, Lea Jessica Flitsch ${ }^{1+}{ }^{0}$, Tamara Krutenko ${ }^{1}$, Pascal Röderer ${ }^{2}$, Liubov Sokhranyaeva ${ }^{3}$, \\ Vira lefremova', Mohamad Hajo ${ }^{1}$, Michael Peitz ${ }^{1,2,4}$ (D), Martin Karl Schwarz ${ }^{2,3}$ and Oliver Brüstle ${ }^{1 *}$ (D)
}

\begin{abstract}
The controlled differentiation of pluripotent stem cells (PSCs) into neurons and glia offers a unique opportunity to study early stages of human central nervous system development under controlled conditions in vitro. With the advent of cell reprogramming and the possibility to generate induced pluripotent stem cells (iPSCs) from any individual in a scalable manner, these studies can be extended to a disease- and patient-specific level. Autism spectrum disorder (ASD) is considered a neurodevelopmental disorder, with substantial evidence pointing to early alterations in neurogenesis and network formation as key pathogenic drivers. For that reason, ASD represents an ideal candidate for stem cell-based disease modeling. Here, we provide a concise review on recent advances in the field of human iPSC-based modeling of syndromic and non-syndromic forms of ASD, with a particular focus on studies addressing neuronal dysfunction and altered connectivity. We further discuss recent efforts to translate stem cell-based disease modeling to 3D via brain organoid and cell transplantation approaches, which enable the investigation of disease mechanisms in a tissue-like context. Finally, we describe advanced tools facilitating the assessment of altered neuronal function, comment on the relevance of iPSC-based models for the assessment of pharmaceutical therapies and outline potential future routes in stem cell-based ASD research.
\end{abstract}

Keywords: Autism spectrum disorder, Induced pluripotent stem cells, Cell reprogramming, Brain organoids, Neuronal connectivity, In vitro differentiation

\section{Introduction}

According to the 5th edition of the 'Diagnostic and Statistical Manual of Mental Disorders', autism is a developmental disorder with impairments in social interaction and communication, which is characterized by restricted and repetitive behavior patterns [1]. Autism has an average worldwide prevalence of $0.6-1 \%$. In about $5 \%$ of all

\footnotetext{
*Correspondence: brustle@uni-bonn.de

${ }^{\dagger}$ Arquimedes Cheffer and Lea Jessica Flitsch contributed equally to this work

${ }^{1}$ Institute of Reconstructive Neurobiology, University of Bonn Medical Faculty \& University Hospital Bonn, Venusberg-Campus 1, Building 76, 53127 Bonn, Germany

Full list of author information is available at the end of the article
}

cases, it is associated with syndromic forms of ASD such as fragile X syndrome (FXS; FMR1 mutation), Rett syndrome (RTS; $M E C P 2$ mutation), Angelman syndrome (15q11q13 deletion), Timothy syndrome (CACNA1C mutation), Phelan-McDermid syndrome (22q13 deletion) and Kleefstra syndrome (EHMT1 mutation) [2]. Furthermore, there are several other genetic conditions, which are less stringently correlated to autism (reviewed in detail by [3]). Interestingly, genes related to synaptic transmission such as NLGN3, NLGN4, NRXN1 and SHANK3 have been shown to play an important role in the pathophysiology of non-syndromic forms of autism [4]. In addition, diverse environmental factors are considered to contribute to the development of the disease [5]. original author(s) and the source, provide a link to the Creative Commons licence, and indicate if changes were made. The images or other third party material in this article are included in the article's Creative Commons licence, unless indicated otherwise in a credit line to the material. If material is not included in the article's Creative Commons licence and your intended use is not permitted by statutory regulation or exceeds the permitted use, you will need to obtain permission directly from the copyright holder. To view a copy of this licence, visit http://creativecommons.org/licenses/by/4.0/. The Creative Commons Public Domain Dedication waiver (http://creativeco mmons.org/publicdomain/zero/1.0/) applies to the data made available in this article, unless otherwise stated in a credit line to the data. 
Since the clinical manifestations and pathological mechanisms of autism are highly heterogeneous, the disease is today referred to as autism spectrum disorder (ASD) (as is more comprehensively outlined in the review by [6]).

Despite the fact that different mechanisms might be involved in the pathogenesis of ASD, there is a general consensus and strong body of evidence that this disorder has a developmental origin. More than $30 \%$ of all pediatric ASD patients carry variants in genes that had previously been associated with developmental delay [7, 8]. Genetic studies in the 1990s revealed that genes affected in FXS and RTS (i.e., FMR1 and MECP2) have important functions during nervous system development $[9,10]$. For example, FMR1 controls the expression of the neural genes SOX1 and PAX6 and is considered highly relevant for neuronal differentiation [11]. In animal models of FXS, specifically fast-spiking GABAergic neurons are affected by a maturation deficit: Compared to control animals, Fmr1 knockout (KO) mice exhibit GABAergic neurons with shorter and less branched dendrites, lower membrane capacitance and increased input resistance, alterations which indicate a delay in the functional maturation of this neuronal subtype [12]. Furthermore, hippocampal neurons of Fmr1-deficient mice show decreased expression of GABA receptor subunits, and the pharmacological blockade of mGluR5 is able to revert the animals' learning and memory deficits as well as increased startle response $[13,14]$. Concordant with this finding, Fmr1 KO mice were reported to show an increase in the number of glutamatergic synapses in specific brain regions, such as the cortex and the hippocampus $[15,16]$.

In case of RTS, glutamatergic hyperfunction seems to play an important role, too, since treatment of mice deficient for the 5mC-binding transcription factor Mecp2 with the mGluR5 negative allosteric modulator CPET and the NMDAR antagonist ketamine reduces RTS-associated phenotypes such as deficits in cognition and information processing, respectively $[17,18]$. Lack of Mecp2 was further found to interfere with the cell cycle dynamics of neural progenitor cells (NPCs) and their transition into more mature stages, as indicated by the delayed maturation of cortical neurons observed in Mecp2-deficient mice [19]. Moreover, Mecp 2 KO results in impaired dendritic arborization as well as deficiencies in synapse formation and network integration of newborn neurons in the adult mouse hippocampus [20].

Together, these finding suggest that alterations in glutamatergic and GABAergic neurons and thus excitation-inhibition (E/I) balance might be one of the key pathogenic mechanisms underlying ASD. In some ASD cases E/I imbalance might also result from impaired glutamatergic neurotransmission, since in a recent study loss-of-function mutations in the AMPAR gene GRIA2 were found in 8 out of 28 patients diagnosed with ASD or ASD-related phenotypes [21]. Furthermore, mutations in GRIA3 and NMDAR-coding genes (e.g., de novo mutations in GRIN2A, truncating mutations in GRIN2C, GRIN3A and GRIN3B and GRIN2B gain-of-function mutations) as well as alterations in metabotropic receptor subunits, especially GRM1 and GRM5, have been linked to psychiatric and neurodevelopmental disorders including ASD [22].

Neuroimaging and post mortem studies provide additional evidence for altered neural connectivity in ASD patient brains, reinforcing the assumption that a neurodevelopmental component and especially an evolving $\mathrm{E} / \mathrm{I}$ imbalance are integral parts of ASD pathogenesis. Structural magnetic resonance imaging (MRI) has revealed that in ASD, brain overgrowth during infancy is followed by a relative decrease in brain size of the frontal and temporal cortices during pre-adolescence and adulthood [23, 24], and functional MRI scans in adult ASD patients hint to neuronal hyperconnectivity between cortical and subcortical regions such as amygdala and thalamus [25]. Post mortem studies have revealed higher spine densities on glutamatergic cortical projection neurons of non-syndromic ASD patients [26]. Vice versa, patients with Angelman syndrome were reported to exhibit reduced cortical expression of $\mathrm{GABA}_{\mathrm{A}}$ receptor [27]. Morin-Parent et al. recently found that FXS patients show reduced activity of GABAergic neurons, resulting in cortical hyperexcitability [28]. Interestingly, brain MRI scans of macaques carrying a mutation in SHANK3 revealed region-specific hyper- as well as hypoconnectivities: While hyperconnectivity was evident in the somatosensory cortex, extrastriate cortical areas and the posterior cingulate cortex, other brain regions such as the medial prefrontal cortex, thalamus, striatum and motor regions were hypoconnected. These complex deviations in neuronal connectivity were associated with behavioral abnormalities characteristic for ASD in humans such as repetitive behaviors as well as motor, social and learning deficits [29].

Considering a neurodevelopmental origin of ASD pathogenesis, the aim of this review is to provide a concise summary of the recent advances and findings in the field of induced pluripotent stem cell (iPSC)-based modeling of ASD. Since iPSC models are widely used to mimic key aspects of early human (neuro)development, they are considered particularly useful for investigating ASD-related alterations in neuronal function and connectivity. 


\section{Cell reprogramming and genome editing as basis for disease modeling}

The iPSC technology provides a unique opportunity to reprogram somatic cells from healthy or diseased individuals to an embryonic stem cell (ESC)-like stage [30, 31]. Thus, iPSCs represent an expandable cellular resource for the derivation of a huge variety of somatic cell types, which can be used for developmental studies, disease modelling and pharmaceutical compound screening in vitro. In the context of brain research, human pluripotent stem cells (PSCs) have opened an alternative route to generate otherwise inaccessible patient-specific neural cells in virtually unlimited numbers in vitro. The validity and applicability of PSC-based models depend heavily on the quality of the PSC-derived neural cultures [32]. Most importantly, PSCs and their derivatives have to be rigidly quality controlled for genomic integrity, for instance by comparative genomic hybridization, single nucleotide polymorphism (SNP) analysis and prospectively also exome or whole genome sequencing [33]. In order to address topics such as E/I imbalance, neuronal differentiation of PSCs has to be highly standardized. For example, it has been shown that even very small fluctuations in the fraction of GABAergic neurons can have profound effects on the overall in vitro network activity [34].

Traditionally, extrinsic factor-guided protocols have been used to drive neuronal differentiation of PSCs. For the generation of cultures highly enriched for excitatory cortical projection neurons, the group of Frederick Livesey published a three-step protocol starting with the directed differentiation of PSCs into cortical precursors by combining dual SMAD inhibition [35] with activation of the retinoid signaling pathway [36]. These cortical precursors subsequently undergo functional maturation and form neuronal networks consisting of approximately equal proportions of upper and deep layer excitatory cortical neurons [36]. Further addition of the MEK/ERK inhibitor PD0325901 and the gammasecretase/notch-signaling inhibitor DAPT results in almost pure neuronal cultures consisting of approximately $90 \%$ projection neurons staining positive for either the cortical layer V marker CTIP2 or the cortical layer VI marker TBR1 after 8 weeks of differentiation [37]. With this system, functional human autapses exhibiting electrophysiological properties comparable to those of primary mouse cortical neurons can be established [38]. In addition to the development of protocols generating excitatory cortical neurons, strategies for the differentiation of cortical inhibitory interneurons were published. In 2013, two independent groups revealed that activation of FOXG1 and sonic hedgehog signaling via a combination of dual SMAD inhibition with the tankyrase inhibitor XAV939 and SHH induces a ventral telencephalic fate in ESCs resulting in efficient generation of medial ganglionic eminence-like precursors $[39,40]$. After a 30-day long co-culture with primary mouse cortical pyramidal neurons [34] and glial cells [35], approximately $80 \%$ of the neurons derived by these protocols displayed characteristics of functional GABAergic neurons.

Although these protocols yield substantially enriched cultures of specific neuronal subtypes relevant for ASD and might even partially recapitulate in vivo neurodevelopment [36], they usually require extended periods of time to achieve cell fate specification and functional maturation, thereby limiting their applicability for standardized neuronal connectivity studies. For this purpose, other approaches such as forward programming, which refers to transcription factor-facilitated differentiation of PSCs towards a specific cell fate, might be of great use (Fig. 1). In 2013, the groups of Thomas Südhof and Marius Wernig reported a protocol for the rapid induction of functional cortical excitatory neurons from PSCs by lentiviral overexpression of the single transcription factor NGN2 [41]. A few years later, the same groups suggested a similar method for the generation of GABAergic neurons using transient overexpression of ASCL1 and DLX2 [42]. We employed genome editing to refine these protocols by expressing the transcription factors in an inducible manner from the human AAVS1 genomic safe harbor locus. This approach results in improved homogeneity and standardization of the induced neuronal cultures, yielding up to $90 \%$ of VGLUT2-positive and GABA-expressing neurons after overexpression of NGN2 or ASCL1 plus DLX2, respectively [38, 43].

The fact that PSCs are easily amenable to genome editing can be exploited for disease modeling by generating isogenic controls, either by repairing existing diseasecausing mutations in patient lines or by newly introducing mutations in healthy control cell lines, mimicking genetic disease preconditions (Fig. 1). This strategy might be particularly relevant for modeling genetic variants with rather subtle effect size [44], since it isolates the effects of a specific mutation and is not biased by additional interindividual differences present in non-isogenic comparisons. Indeed, a number of mutations discussed in the context of ASD show low penetrance [45]. While the exact reasons for this phenomenon can be manifold, it is conceivable that in many cases low penetrance mutations elicit rather subtle changes in neuronal functioning, which become symptomatic only in combination with additional predisposing factors. Here, genome editingbased studies on an isogenic background can increase the resolution of subtle pathogenotypes, although modeling of low effect sizes in PSC-based systems remains a challenge. 


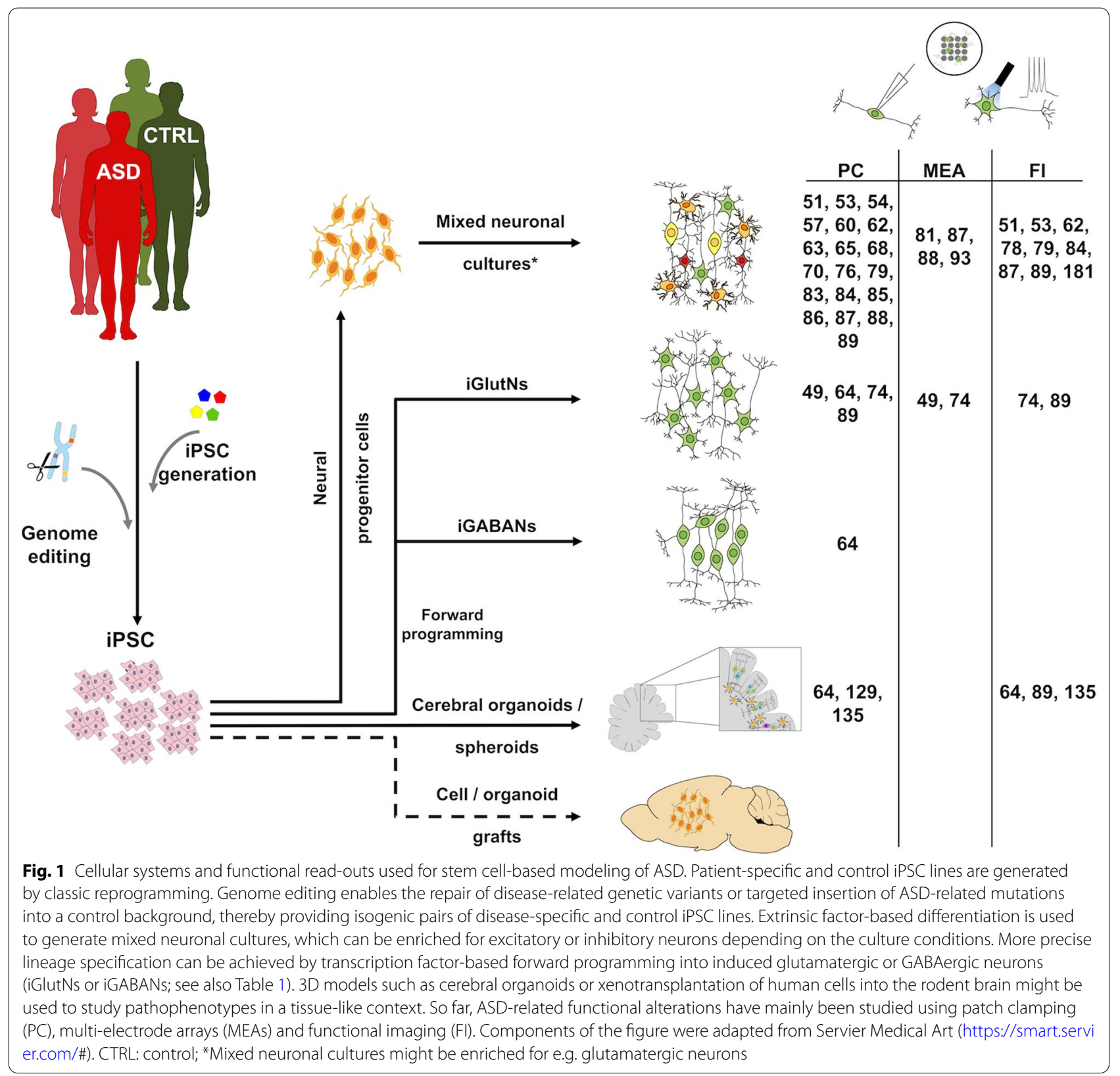

Notably, CRISPR/Cas9-based genome editing has already been successfully used for modeling ASD. For example, a recent study addressed the role of the CREB pathway-which has been reported to directly regulate the expression of genes related to dendritic arborization [46] and indirectly influences MECP2 levels [47]-in RTS using a portfolio of ESC and iPSC lines comprising a patient-specific $M E C P 2$ mutant line, a CRISPR/Cas9-corrected patient line and a control cell line in which $M E C P 2$ was knocked out [48]. In another study by Deneault et al., CRISPR/Cas9 was used to establish 10 isogenic iPSC lines, each carrying KOs of different ASD-relevant genes, with the aim to reveal common disease-related phenotypes [49]. The results of this approach are depicted in more detail in the next paragraph. An additional asset of using genome-edited PSCs for disease modeling is the feasibility to mimic polygenic diseases by simultaneously inducing or repressing the expression of multiple diseaseassociated genes in one cell line. This can be achieved by the use of multiple guide RNAs directing complexes of inactive Cas9 mutants fused to transcriptional activators or repressors to their respective DNA target sites. Schrode et al. used this approach to simultaneously modulate the expression of 4 different genes (FURIN, 
SNAP91, TSNARE1 and CLCN3), for which SNPs have been reported to be related to schizophrenia (SCZ) [50]. Thus, this concept might also be informative for investigating non-syndromic forms of ASD or for probing low penetrance mutations in a defined genetic background harboring already other disease-related variants.

\section{ASD-related phenotypes in 2D models}

In general, alterations in neuronal differentiation and maturation have the potential to elicit functional consequences. Indeed, human neurons derived from patient ASD iPSCs or PSCs engineered to carry ASD-related genetic variants have been reported to display a number of changes in their intrinsic properties, which can be expected to alter connectivity and network activity (Table 1).

\section{Functional phenotypes detected in iPSC-based models of FXS and RTS}

An early study based on FXS ESCs revealed that the disease-associated lack of FMR1 expression leads to the derivation of NPCs with impaired neuronal maturation and increased gliogenic potential. Although FXS neurons were able to generate action potentials, they showed little spontaneous synaptic activity and no responsiveness to glutamate [11]. Achuta et al. identified various functional alterations in FXS iPSC-derived neural progenitors, including an increased differentiation of cells expressing AMPA receptors. The cells exhibited augmented intracellular calcium responses to AMPA and kainate that were mediated through calcium-permeable AMPARs lacking the GLUA2 subunit, which is encoded by GRIA2. The authors discuss that this could be due to a scenario where the loss of the RNA-binding protein FMRP leads to increased levels or mislocalization of the GRIA2repressive microRNA miR-181a [51]. In addition, FXS iPSC-derived neurons generated in another study were found to show hsa-miR-382-dependent elevated REST levels leading to a downregulation of genes involved in axon pathfinding such as ROBO3, DCC and SLIT1 [52].

RTS patient iPSC-derived neurons, too, exhibit morphological abnormalities such as a reduced soma size and decreased synaptic density, as well as functional deficits including altered calcium signaling, a decreased frequency of excitatory postsynaptic currents (EPSCs) [53] and a delayed GABA functional switch from excitation to inhibition, which is due to decreased expression of the MECP2 target gene KCC2 [54]. An iPSC-based model of RTS was further used to analyze the potency of selected compounds to revert the mutation-associated reduction in MECP2 protein levels. More specifically, the authors tested for the effect of low dosages of gentamicin and treatment with IGF1 [53]. In prokaryotes, the aminoglycoside gentamicin possesses the ability to suppress premature stop mutations by binding $16 \mathrm{~S}$ rRNA, thus impairing ribosomal proofreading. In eukaryotic cells, gentamicin was shown to induce low levels of translational misreading, too [55]. In human RTS-derived neuronal cultures, gentamicin treatment increased MECP2 protein levels and rescued the number of glutamatergic synapses [53]. IGF1 treatment was reported to partially alleviate RTS-like symptoms in Mecp2-deficient mice [56] and resulted in increased numbers of glutamatergic synapses on RTS patient-derived neurons, too [53]. Interestingly, even mutations affecting only one of the two main MECP2 isoforms are sufficient to provoke RTS-associated phenotypes: MECP2e1 isoform-deficient patient iPSC-derived neurons were reported to show reduced soma size, less complex dendritic arborization, increased input resistance, reduced membrane capacitance and lower frequency of action potential firing [57]. In contrast to RTS iPSC models, which are associated with a downregulation of MECP2, an increased number of synaptic puncta and synchronous bursting activity were detected in a model of $M E C P 2$ duplication [58]. IPSC-derived neurons from patients with mutations in $C D K L 5$, which typically present with a clinical phenotype similar to RTS [59], also display a reduced number of excitatory synapses with aberrant spine structure [60]. It remains to be investigated, however, whether these changes result from similar pathogenic mechanisms.

\section{Neuronal dysfunction in stem cell models of other syndromic forms of ASD}

Angelman syndrome, which is caused by a deletion of the maternal 15q11-q13 chromosomal region, results in a complete lack of $U B E 3 A$ expression, since the paternal allele encoding for this ubiquitin protein ligase is normally not expressed due to genomic imprinting [61]. In 2017, Fink et al. demonstrated that neurons differentiated from Angelman syndrome patient iPSCs via an extrinsic factor-guided neuronal differentiation protocol exhibit impaired neuronal maturation, as characterized by a more depolarized resting membrane potential and a reduced proportion of neurons firing mature action potentials, as well as decreased excitatory synaptic activity and a lower capacity for activitydependent synaptic plasticity [62]. Vice versa, neurons differentiated from iPSCs of patients with duplication of the Angelman syndrome-affected chromosomal region exhibited higher EPSC amplitudes and frequencies [63]. In another recent study, the consequences of $U B E 3 A$ mutations were assessed using NGN2-induced patient neurons and brain organoids. Here, the authors found that calcium- and voltage-dependent big potassium (BK) channels undergo UBE3A-mediated ubiquitination and 


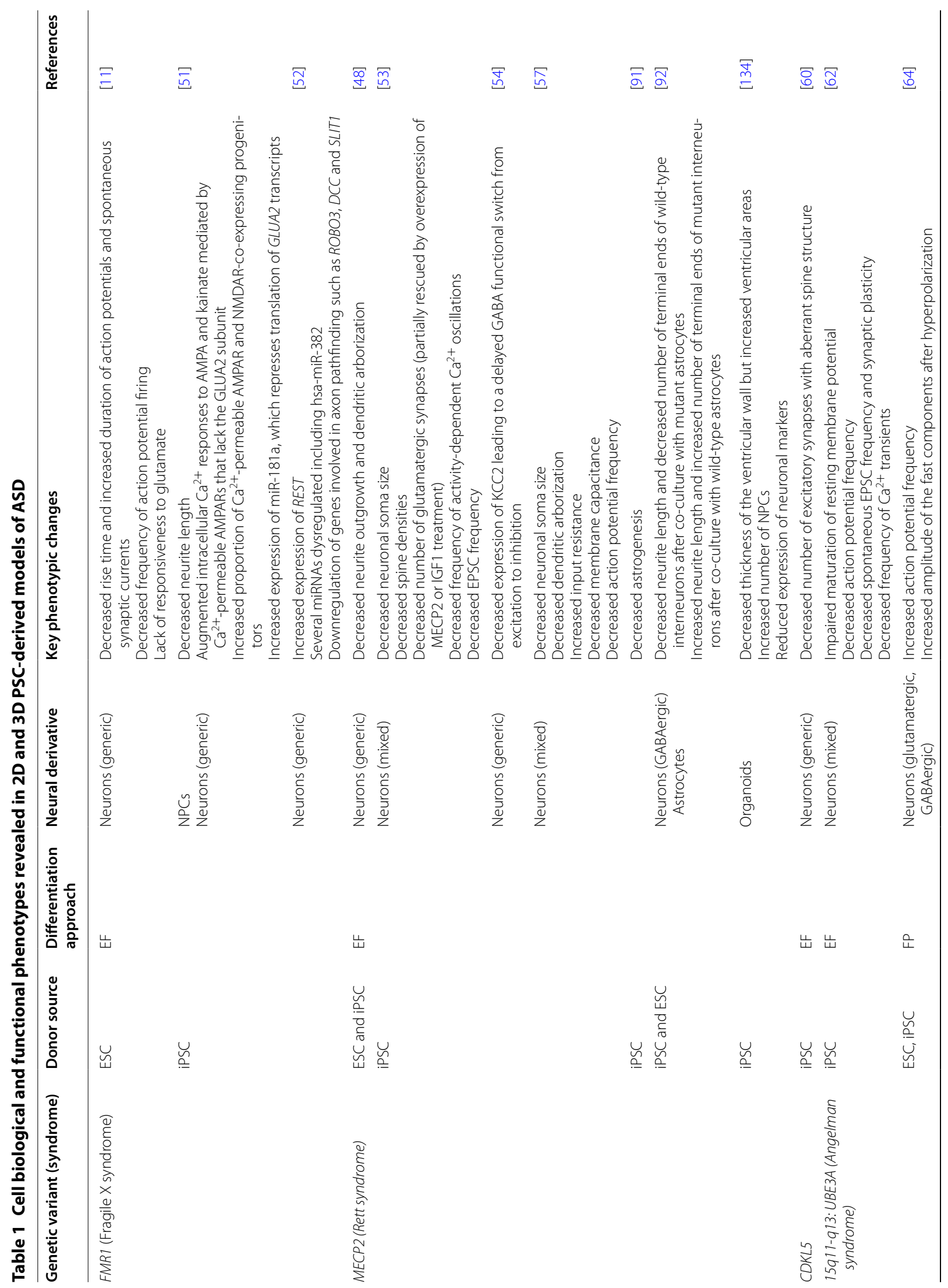




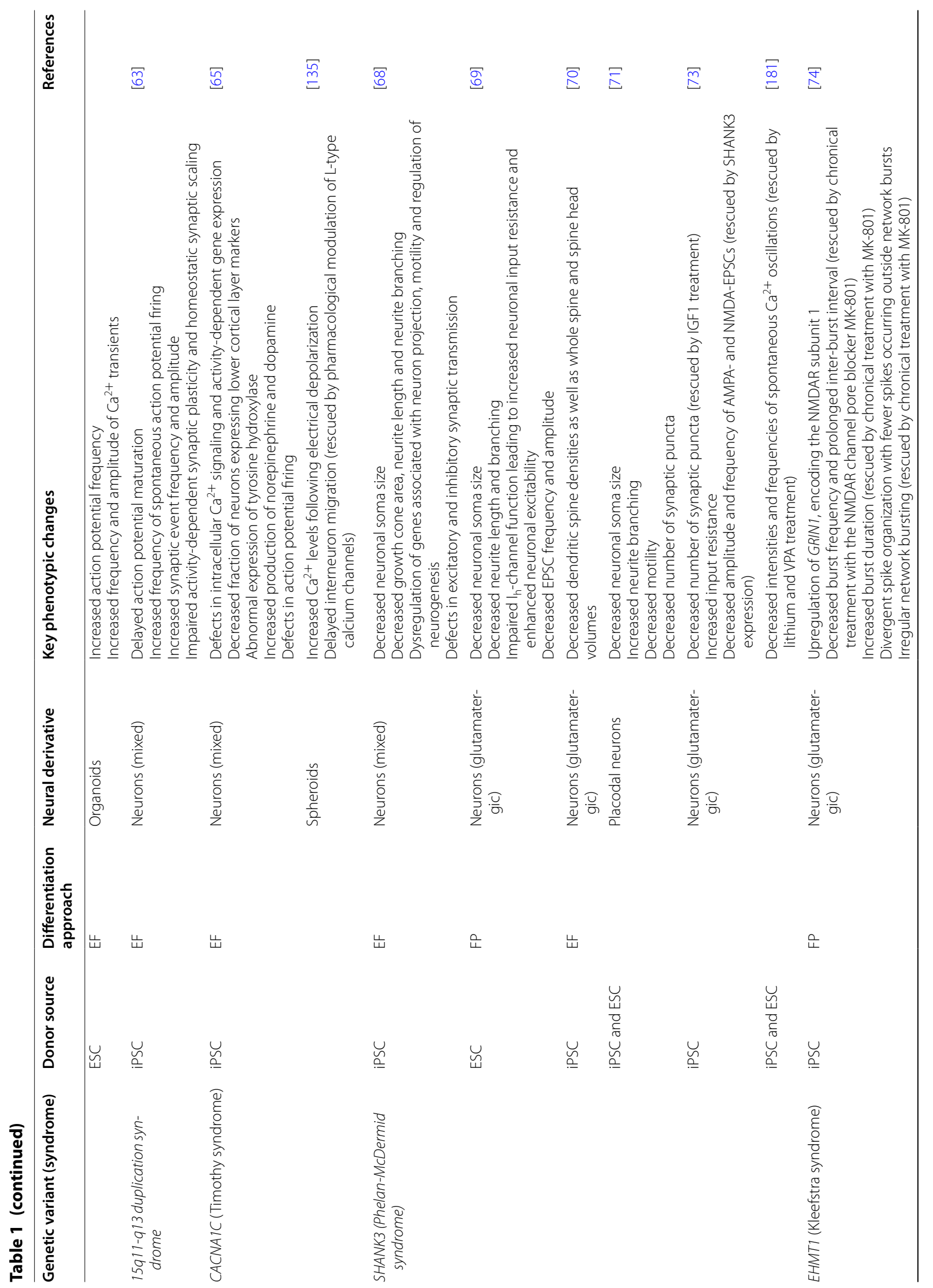




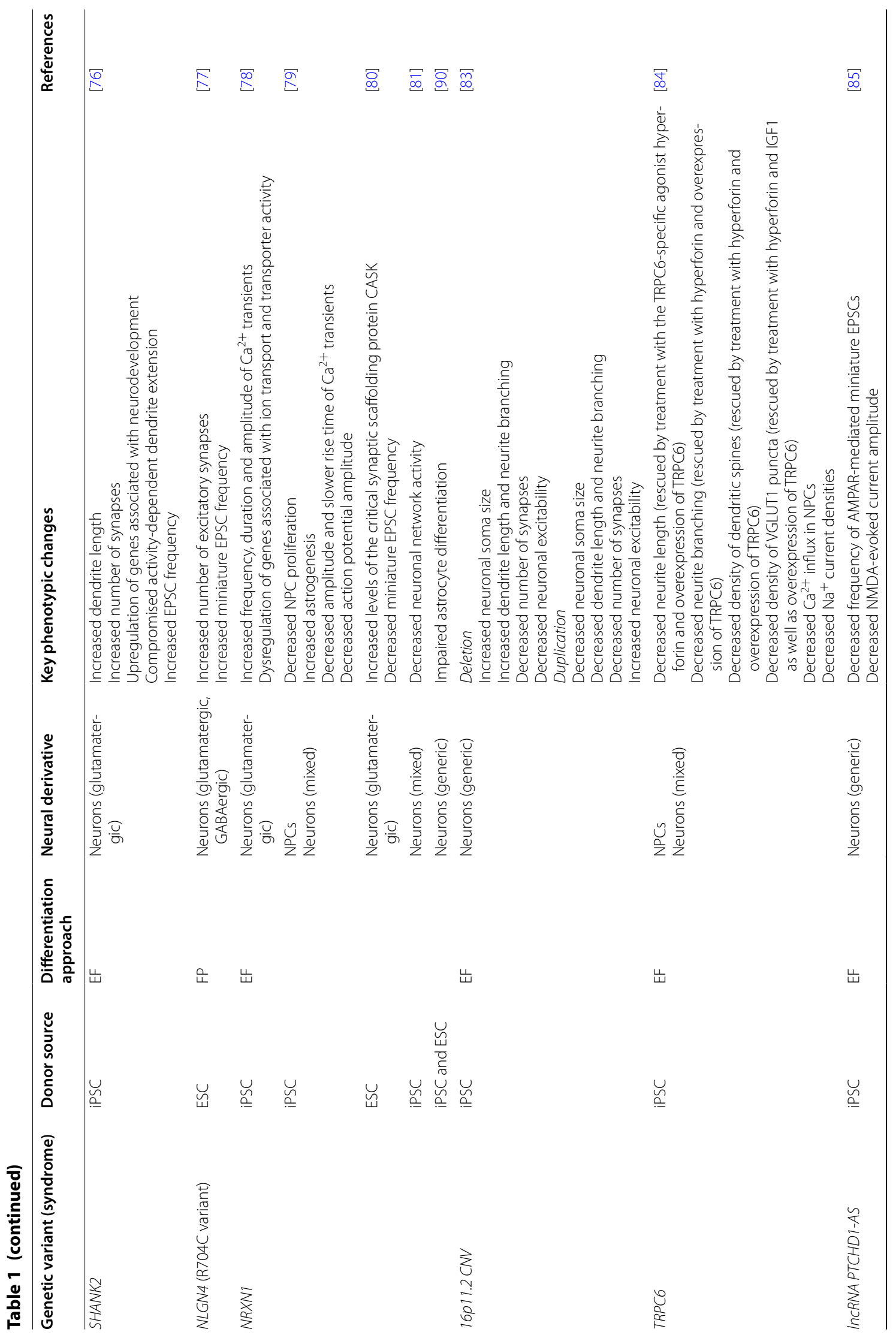




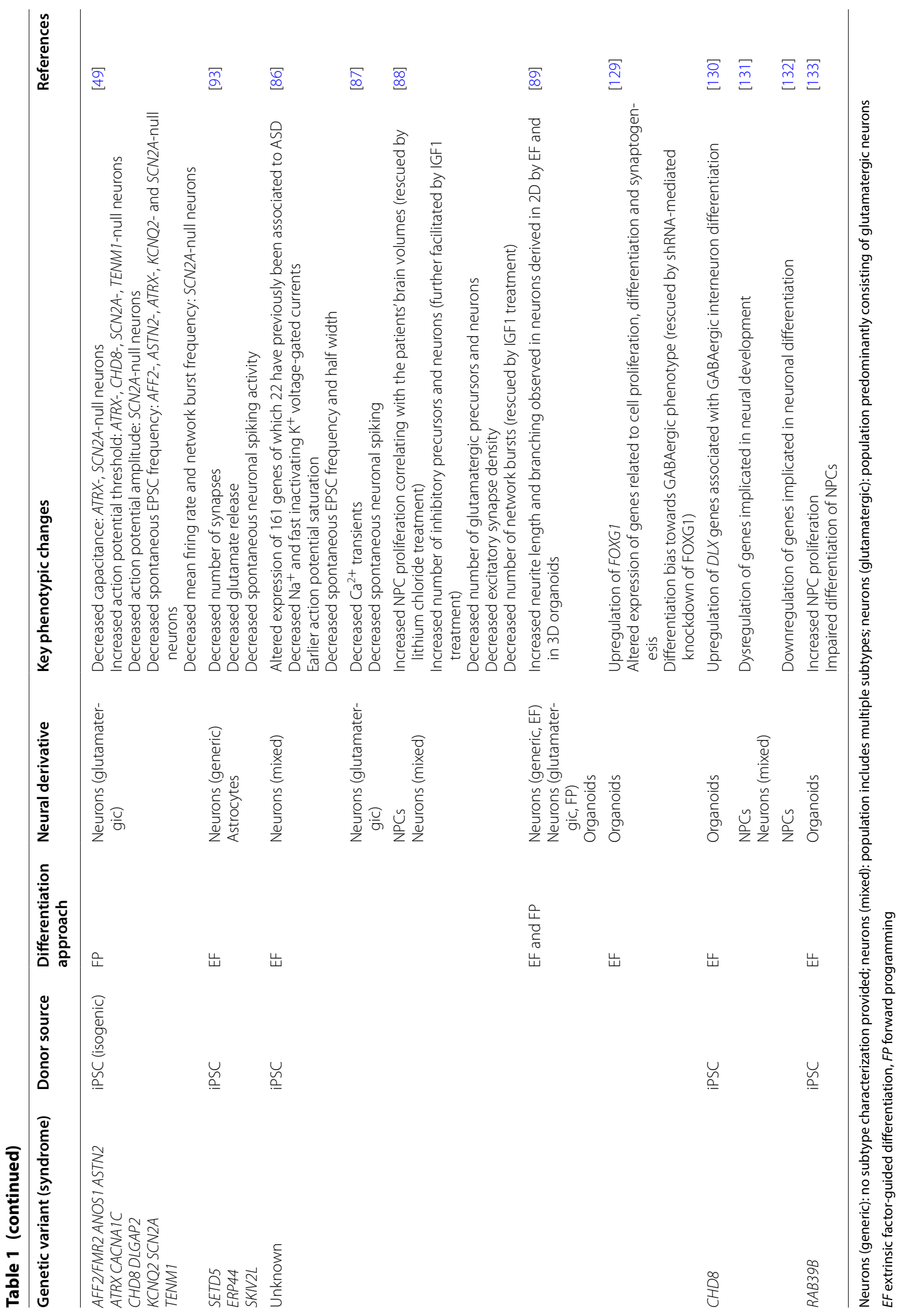


subsequent proteasomal degradation. Consequently, loss of UBE3A leads to an increased activity of BK channels, which in turn causes neuronal hyperexcitability along with network synchronization. Indeed, antagonists for the affected BK channel could normalize neuronal excitability, pointing to BK channels as potential drug targets for the treatment of Angelman syndrome patients [64].

Despite the many advantages of protocols that are highly tailored towards the generation of a specific PSCderived neuronal subtype, it might be still informative to use less stringent neuronal differentiation methods, since divergent in vitro neuronal subtype specification might be itself a disease phenotype. Paşca et al., for example, found that neuronal cultures generated from Timothy syndrome iPSCs-carrying mutations in CACNA1C, which encodes for the L-type calcium channel $\mathrm{Ca}_{\mathrm{v}} 1.2-$ exhibit a sustained rise in intracellular calcium after depolarization and an increase in action potential width. Resulting activity-dependent gene expression changes were enriched for genes related to calcium-dependent CREB signaling, including the CREB downstream target $T H$. The authors further revealed that although neuronal differentiation of Timothy syndrome iPSCs mainly gave rise to neurons with cortical identity, these cells showed abnormally high expression levels of tyrosine hydroxylase and secreted more catecholamines such as dopamine and norepinephrine than control neurons. Treating patient iPSC-derived neurons with the L-type calcium channel modulator roscovitine decreased the number of tyrosine hydroxylase-positive neurons [65].

Another well-established genetic variant for ASD is SHANK3 deficiency, which is associated with PhelanMcDermid syndrome [66]. SHANK3 belongs to a family of adaptor proteins which interact with the actin cytoskeleton and different components of the postsynaptic density, and thus plays an important role in synapse development and function [67]. SHANK3 $\mathrm{KO}$ in PSCderived neurons results in decreased neuronal soma size, reduced neurite length and less neurite branching [68, 69], and a recent publication focusing on patients with de novo mutations in SHANK3 reports decreased dendritic spine densities and volumes in SHANK3-mutated neurons [70]. In another study, placodal but not cortical neurons differentiated from iPSCs of ASD patients carrying microdeletions in the SHANK3 locus and from SHANK3 KO ESCs were found to have smaller cell somata and fewer synapses compared to control neurons, too, but in this study more extensive neurite branching has been observed [71]. Focusing on functional alterations, Thomas Südhof's group observed an increased input resistance leading to augmented excitability in neurons derived from ESCs with conditional homozygous or heterozygous SHANK3 loss-of-function mutations. In their elegant study, the authors revealed that the hyperexcitability of SHANK3-deficient human neurons is caused by a significant impairment of hyperpolarization-activated cation $\left(\mathrm{I}_{\mathrm{h}}\right)$ channels, which was accompanied by a decreased frequency and amplitude of EPSCs. Their work suggests that SHANK3 interacts with hyperpolarizationactivated cyclic nucleotide-gated $(\mathrm{HCN})$ proteins, which generate $\mathrm{I}_{\mathrm{h}}$ channels. SHANK3 inactivation might thus contribute to the ASD pathogenesis via induction of an $I_{h}$ channelopathy [69]. Notably, forebrain excitatory neurons derived from Phelan-McDermid syndrome patient iPSCs, which carry heterozygous 22q13.3 deletions comprising the SHANK3 gene, were shown to exhibit an increased input resistance and decreased EPSC frequency and amplitude, too. These phenotypic changes selectively affected excitatory transmission, as no alterations in the amplitude and frequency of inhibitory postsynaptic currents were found. In this model, IGF1 treatment was demonstrated to increase the number of synapses that lacked SHANK3 but contained PSD95 and NMDARs with fast deactivation kinetics. Since such synapses showed rapidly decaying EPSCs-similar to synapses that appear in later stages of neurodevelopment [72] - the authors hypothesized that IGF1 rescues synaptic activity in Phelan-McDermid syndrome patientderived neurons by promoting synapse maturation [73].

Finally, decreased enzymatic activity of the histone methyltransferase EHMT1, which is affected by heterozygous loss-of-function mutations associated to the ASD-related Kleefstra syndrome, was reported to result in decreased H3K9me2 levels at the promoter of the GRIN1 gene, which encodes the NMDAR subunit 1 . The consequently increased NMDAR/AMPAR ratio caused significant aberrations in neuronal network activity of EHMT1 haploinsufficient neurons, which exhibited less regular network bursting as well as an overall decrease in burst frequency and an increase in burst duration. Inhibition of NMDARs by D-AP5 transiently ameliorated the NMDAR/AMPAR imbalance by driving the incorporation of AMPARs, and chronic treatment of EHMT1deficient neurons with the NMDAR channel pore blocker MK-801 for 7 days even partially normalized network activity [74].

\section{Functional phenotypes in stem cell models of non-syndromic and idiopathic ASD}

Interestingly, iPSC-derived neurons from patients with SHANK2 haploinsufficiency, another member of the family of SHANK genes involved in ASD [75], displayed dendrite abnormalities and increased synapse numbers as well as a higher spontaneous EPSC frequency leading to a hyperconnected phenotype of SHANK2-deficient neurons [76]. Mutations in NLGN4, which encodes for a 
neuronal cell surface protein, too, might result in more active neuronal networks, since an increased number of excitatory synapses and higher frequency of miniature EPSCs have been reported for neurons differentiated from ESCs engineered to carry the ASD-associated R704C mutation in NLGN4 [77]. Different results have been communicated regarding the functional consequences of mutations in NRXN1, which encodes for a presynaptic membrane cell adhesion protein. Avazzadeh et al. demonstrated that glutamatergic cortical neurons derived from $N R X N 1 \alpha^{+/-}$iPSC lines exhibit increased frequency, duration and amplitude of calcium transients in comparison to control neurons derived from healthy donors [78]. On the other hand, neurons differentiated from patient iPSCs with biallelic NRXN1 $\alpha$ deletion displayed a decreased amplitude and slower rise time of depolarization-evoked calcium transients, as well as a reduction in action potential amplitude [79]. In line with this, an earlier study by Pak et al. reported that glutamatergic neurons differentiated from engineered ESCs carrying heterozygous loss-of-function mutations in NRXN1 show lower frequencies of miniature EPSCs compared to isogenic controls [80], and Kristen Brennand's group, too, observed reduced neuronal network activity of glutamatergic iPSC-derived neurons with heterozygous intragenic NRXN1 deletions. Specifically, the authors assessed two rare deletions in the $3^{\prime}$ region of the NRXN1 locus and found that they affect NRXN1 splicing, resulting in the expression of mutant $N R X N 1 \alpha$ isoforms, which were not identified in neurons derived from either healthy controls or patients carrying deletions in the $5^{\prime}$ region of NRXN1. Interestingly, overexpression of 4 different wild-type NRXN1 $\alpha$ isoforms could rescue the reduced neuronal network activity observed in $5^{\prime}-\mathrm{NRXN1} 1^{+/-}$neurons, whereas it did not functionally impact $3^{\prime}-\mathrm{NRXN1}^{+/-}$neurons. Overexpression of two of the newly identified mutant isoforms decreased the activity of neurons derived from controls and $5^{\prime}$-NRXN1 ${ }^{+/-}$carriers. This genotype-dependent mode of action of NRXN1 $\alpha$ isoforms suggests the existence of a dominant-negative effect for specific heterozygous NRXN1 deletions in the pathogenesis of NRXN1-related psychiatric disorders including ASD. Lastly, single-cell RNA sequencing analysis further revealed that besides exhibiting synaptic dysfunction, patient iPSC-derived neurons were underrepresented in cell clusters consisting of mature neurons. This suggests that NRXN1 mutations might impair neuronal maturation, too [81].

Notable observations have also been made in the context of aberrations affecting the 16p11.2 chromosomal region, for which deletions as well as duplications have been associated with ASD [82]. Although penetrance of these mutations can be highly variable [45], Deshpande et al. found that iPSC-derived cortical neurons from deletion carriers showed an increase in neuronal soma size and dendrite length, which were associated with decreased excitability. In contrast, neurons with 16p11.2 duplication showed opposite morphological alterations (i.e., reduced cell size and dendrite length). These observations might at least partially explain the macro- and microcephalic phenotypes observed in 16p11.2 deletion and duplication carriers, respectively. Interestingly, neurons from both genotypes exhibited a reduction in synaptic density, which would be in line with common behavioral symptoms observed in 16p11.2 CNV carriers [83].

In 2015, Griesi-Oliveira et al. identified the ion channel coding gene TRPC6 as a new candidate involved in non-syndromic ASD. Comparing control iPSC-derived NPCs and neurons with those of one ASD-affected individual with TRPC6 haploinsufficiency, the authors found reduced calcium influx in NPCs and impaired neuronal maturation, as indicated by shorter and less branched neurites, as well as a reduction in the density of dendritic spines and VGLUT1-positive synaptic puncta. The observed neuronal phenotypes could be partially reverted by overexpression of TRPC6 or treatment with the TRPC6 agonist hyperforin. Importantly, the authors further demonstrated that the RTS-associated MECP2 acts as a positive transcriptional regulator of TRPC6 expression, and IGF1- which is able to revert the decrease of glutamatergic synapses in MECP2-mutant RTS-derived neurons [53] and SHANK3-mutant Phelan-McDermid syndrome-derived neurons [73]-rescued glutamatergic synapse numbers in TRPC6-mutant neurons as well [84].

Functional impairments in excitatory neurotransmission, characterized by a decreased frequency of AMPARmediated miniature EPSCs and a reduced amplitude of ionic currents elicited by NMDARs, have also been reported for neurons differentiated from patient iPSCs carrying (micro)deletions in the Xp22.11 chromosomal region affecting gene loci encoding the lncRNA PTCHD1-AS [85].

Using NGN2-based forward programming into glutamatergic neurons and CRISPR/Cas9-based gene inactivation, Deneault et al. investigated the effect of 10 additional ASD-related genes (i.e., AFF2/FMR2, ANOS1, ASTN2, ATRX, CACNA1C, CHD8, DLGAP2, KCNQ2, $S C N 2 A$ and TENM1) on neuronal function. They found that $\mathrm{KO}$ of either of the genes AFF2/FMR2, ASTN2, $A T R X, K C N Q 2$ and $S C N 2 A$ significantly reduced spontaneous EPSC frequency in iPSC-derived excitatory neurons. An increased action potential threshold was observed in ATRX-, CHD8-, SCN2A- and TENM1-KO neurons; ATRX- and $S C N 2 A-\mathrm{KO}$ neurons additionally exhibited decreased capacitance. Interestingly, $\mathrm{KO}$ 
of $S C N 2 A$ further led to a reduction in action potential amplitude, mean firing rate and network burst activity [49].

Some of these results fit well with data generated with iPSCs from idiopathic ASD patients. For example, Liu et al. generated iPSC-derived neurons from 3 idiopathic male ASD patients and their unaffected male siblings, identifying in total 161 differentially expressed genes, of which 22 had previously been associated to ASD as for example NRXN3 and $S C N 2 A$ [86]. This and other studies on idiopathic ASD further revealed several functional alterations in iPSC-derived neurons including decreased calcium transients, decreased sodium and fast inactivating potassium voltage-gated currents, decreased spontaneous neuronal spiking and an earlier action potential saturation, as well as a reduced frequency and half width of spontaneous EPSCs [86, 87]. These findings are paralleled by data pointing to altered neurogenesis: In idiopathic ASD patients with early developmental brain overgrowth, increased proliferation of patient iPSC-derived NPCs positively correlated with the patients' brain volumes and could be reverted by transfecting BRN2 or stabilizing $\beta$-catenin activity via lithium chloride treatment. In addition to altered proliferation, patient iPSC-derived neuronal precursors exhibited a differentiation bias towards inhibitory fates, resulting in an increased fraction of inhibitory neurons and reduced numbers of excitatory neurons with decreased glutamatergic synapse densities. Accordingly, patient-derived neurons produced less neuronal spikes and network bursts, which was successfully reverted by IGF1 treatment [88]. Analyzing the molecular causes of altered neurogenesis in this idiopathic ASD patient cohort in further depth, Schafer et al. assessed regulatory gene networks during neural induction and identified a gene module with different expression dynamics in patient iPSCs versus control cells. ASD neurons showed accelerated progression through this module, resulting in premature differentiation. They also provide evidence that these differences might be caused by increased chromatin accessibility in patient NPCs, leading to aberrant epigenetic imprinting. Interestingly, bypassing the proliferative NPC stage via an NGN2-based forward programming approach indeed normalized neurogenesis from ASD patient-derived iPSCs [89].

Taken together, data from a diverse collection of models reflecting syndromic as well as non-syndromic forms of ASD point to reduced complexity of neuronal morphology and functional alterations that might contribute to altered $\mathrm{E} / \mathrm{I}$ balance, which is considered a key principle in the pathogenesis of ASD. Yet, these functional alterations are not uniform and encompass both hypo- and hyperactive changes. It remains to be determined how these diverse neuronal phenotypes might contribute to the emergence of the pathognomonic symptoms characteristic for this heterogenous neuropsychiatric disorder.

\section{Modelling the role of glia in ASD}

Considering their important functions in the formation and maintenance of neuronal networks, it is conceivable that glial cells contribute to the pathogenesis of ASD. Indeed, iPSC-derived NPCs from an ASD patient with biallelic NRXN1 $\alpha$ deletion proliferated slower and gave rise to an increased fraction of astrocytes upon differentiation [79], whereas shRNA-mediated knockdown of NRXN1 $\alpha$ in PSC-derived NPCs led to impaired astrogenesis [90]. Differentiation of RTS patient iPSC-derived NPCs, too, resulted in a reduced number of astrocytes as compared to the differentiation of control iPSCs, and quantitative proteomic analysis revealed that the impaired astrogenesis was linked to an increased expression of the neurodevelopmental cell fate regulator LIN28 [91]. Williams et al. investigated the interaction between astrocytes and GABAergic interneurons in RTS and found that $M E C P 2$-mutant astrocytes had a detrimental effect on wild-type human interneurons, which showed a decrease in neurite length and terminal ends. Vice versa, wild-type astrocytes had a beneficial effect on patient interneurons resulting in increased neurite lengths and more terminal ends. Interestingly, treatment of mutant astrocyte-neuron co-cultures with either IGF1 or GPE-a peptide containing the first three amino acids of IGF1positively influenced neuronal morphology, whereas it had negative effects on wild-type co-cultures. The authors hypothesize that decreased expression of IGF1 receptor in mutant astrocytes might contribute to the divergent responses observed after treatment [92]. Data from a study by Russo et al. further suggest that iPSCderived astrocytes from non-syndromic ASD patients release increased amounts of reactive oxygen species and IL-6, which then impair neuronal morphology and synapse formation when co-cultured with control iPSC-derived neurons. Vice versa, control iPSC-derived astrocytes improved synaptogenesis of ASD-derived neurons, which were phenotypically characterized by fewer synapses, reduced glutamate release and a decrease in spontaneous neuronal activity. Treatment of mutant astrocytes with anti-IL-6 and control astrocytes with recombinant IL- 6 mimicked the phenotype of the respective other condition [93]. Although studies on the role of non-neuronal cells in the pathogenesis of neuropsychiatric disorders are still at an early stage, these observations from stem cell models suggest that glial cells can significantly contribute to neuronal impairment in ASD. 


\section{Non-genetic factors associated with ASD}

In addition to inherited genetic pre-dispositions, nongenetic parental conditions might increase the offspring's risk for developing a neuropsychiatric disease: Some groups reported that parental age might be positively correlated with the offspring's ASD risk [94, 95]. In addition, maternal infections during pregnancy such as rubella or influenza have been linked to ASD [96-98]. Elevated levels of amniotic estrogens and prenatal exposure to progestin, too, have been reported to generate an increased risk of developing ASD during childhood [99]. The same applies to prolonged prenatal exposure to paracetamol [100-102], antidepressants [103] and valproic acid (VPA) [104]. While prescription of VPA during pregnancy is highly discouraged [105], it continues to serve as a proofof-concept drug in stem cell-based models of developmental neurotoxicity testing (DNT) [106-108]. For example, Miranda et al. treated iPSCs with VPA during neural induction, simulating continuous in vivo exposure during the first 2-3 weeks of gestation. They observed disrupted neural rosette formation and decreased numbers of NCAM-positive cells [107]-neurogenic defects, which could, in principle, provide a basis for functional alterations.

Air pollutants such as nitrogen dioxide and particulate matter, too, have been associated with an increased risk of developing ASD [109-111]. Employing iPSC models, Yamada et al. studied exposure of neural cells to the environmental pollutant tributyltin [112] and the organophosphate and insecticide chlorpyrifos [113], both of which have been linked to an increased risk of ASD in offspring [114, 115]. Their data suggest that both substances lead to reduction of MFN1 and thus to mitochondrial dysfunction, which subsequently impairs neural induction $[112,113]$.

Childhood exposure to heavy metals such as mercury and lead is also considered a risk factor for developing ASD [116, 117]. Raciti et al. demonstrated that exposure of iPSC-derived neural cultures to subtoxic mercury concentrations promotes astrocyte differentiation [118], a phenotype reminiscent to the increased astrogliogenesis observed in ASD-related models of bi-allelic NRXN1 deletions [79].

As in other fields, the use of higher throughput approaches has also been implemented in the context of DNT. Pei et al., for example, employed iPSCs and different iPSC-derived neural cell types such as NPCs, neurons and astrocytes to assess 80 different (developmental) neurotoxicants and environmental pollutants (including, e.g., chlorpyrifos, deltamethrin, diazepam, methyl mercuric (II) chloride, valinomycin and VPA), with 50 of them inducing cytotoxicity in at least one of the assessed cell types [119].
Although stem cell-based studies on DNT have, so far, mostly focused on alterations in neural induction and neurogenesis, it is conceivable that such changes might eventually elicit functional alterations. The fact that various environmental factors are discussed as contributors to ASD clearly warrants further work in this direction. As high throughput applications become increasingly available, such studies might, at some point, also accommodate DNT screens in genetic backgrounds predisposing to ASD.

\section{Prospects for studying ASD-related neuronal dysfunction in organoids}

Classic 2D cultures are easy to grow and facilitate straightforward microscopic image analysis. However, they lack a number of aspects typical for tissues including proper formation of interstitial space and self-organization into organ-specific architectures such as for example the formation of cortical layers. Here, organoids have opened entirely new perspectives, especially for analyzing aspects of organogenesis and developmental defects over prolonged periods of time in vitro (Fig. 1; reviewed in detail by [120-122]). Up to now, however, only few studies have employed 3D systems to model functional alterations in ASD. Some of them relate to DISC1 mutations, which have been identified as a high-risk genetic factor for a wide range of psychiatric disorders including SCZ and ASD [123]. Employing a multifaceted approach to investigate the molecular and cellular role of DISC1 in the pathogenesis of psychiatric disorders, Ye et al. revealed that the $\mathrm{C}$-terminal coiled-coil region of the DISC1 protein is crucial for its interaction with NDE1 and NDEL1. Mutations in DISC1 were shown to result in a disruption of this interaction, leading to reduced NPC proliferation in ventricular zone-like structures of human forebrain organoids [124]. Concordantly, Srikanth et al. found impaired proliferation and structural abnormalities in human cerebral organoids derived from DISC1-mutant iPSCs, which could be rescued by WNT antagonism [125]. Although these organoid studies lack functional data, it is conceivable that reduced NPC proliferation at distinct phases of cortical development might lead to altered neuronal network formation.

Working on dorsal telencephalic organoids generated from idiopathic ASD patients with increased head circumference-a phenotype which has been associated with more severe autism symptoms [126-128]-Mariani et al. detected not only altered expression of genes related to cell proliferation, differentiation and synaptogenesis, but also an accelerated cell cycle and a FOXG1-dependent overproduction of GABAergic neurons [129].

An upregulation of $D L X$ genes involved in GABAergic interneuron differentiation was also observed in 
telencephalic organoids with ASD-associated heterozygous loss of CHD8. Genes differentially expressed in CHD 8 mutant and wild-type organoids were further enriched for pathways relating to neurogenesis, neuronal differentiation, forebrain development, Wnt/ß-catenin signaling and axon guidance [130]. Similar transcriptional changes were previously described for CHD8-mutant 2D cultures of NPCs and immature neurons [131]. Mechanistically, integration of RNA sequencing with genomewide delineation of CHD8 binding in 2D cultures of iPSC-derived NPCs revealed that CHD8-bound genes were strongly associated with chromatin modification and transcriptional regulation, whereas indirectly downregulated genes (i.e., without $\mathrm{CHD} 8$-binding sites) were enriched for pathways involved in brain development, including synapse formation, neuron differentiation, cell adhesion and axon guidance [132].

A very recently published PSC-based KO model of the small GTPase RAB39B, which is associated with macrocephaly and ASD phenotypes, revealed increased proliferation and impaired differentiation of NPCs within human cortical organoids due to upregulated PI3KAKT-mTOR signaling [133]. Larger ventricular zonelike structures as well as impaired neurogenesis have also been described in a 3D cortical model of syndromic RTS [134].

As for the functional consequences of altered neurogenesis, an ESC-based organoid model of Angelman syndrome revealed increased action potential frequency, as well as increased frequency and amplitude of calcium transients, reproducing the same phenotypes that were found in 2D neuronal cultures [64].

Newer models comprising fused organoids representing, for instance, dorsal and ventral forebrain, provide interesting prospects for studying the interplay between cortical excitatory and inhibitory neurons, in particular with respect to the migration and integration of ventrally born GABAergic interneurons into the dorsal cerebral cortex [135-137]. Previous studies in rodents had revealed that the L-type calcium channel $\mathrm{KCC} 2$ regulates interneuron migration by controlling intracellular calcium transients in response to $\mathrm{GABA}_{\mathrm{A}}$ receptor activation [138]. Interestingly, the Timothy syndromeassociated L-type calcium channel $\mathrm{Ca}_{\mathrm{v}} 1.2$ might have similar implications for neuronal migration: Employing live imaging of fused brain spheroids, Sergiu Pașca and colleagues investigated the saltatory migration of GABAergic interneurons, born in ventral forebrain (subpallium) organoids, into dorsal forebrain (pallium) organoids. In this model, Timothy syndrome patient-derived GABAergic interneurons exhibited increased calcium signaling, resulting in an increased saltation frequency and concurrently decreased saltation length and speed, which could be rescued by pharmacologically blocking L-type calcium channels [135]. Again, it seems conceivable that such interneuron migration defects disrupt the tightly orchestrated liaison of inhibitory and excitatory neurons during cortex development and might thus elicit alterations in cortical function.

\section{Cell transplantation and disease modeling in vivo}

Despite their numerous advantages in recapitulating early central nervous system (CNS) tissue formation, cerebral organoids lack a number of structures and cell types encountered in primary CNS tissue. Above all, the lack of a vascular system puts serious constraints on nutrient supply for long-term maintenance. Here, transplantation of human cell suspensions or even organoids into the rodent CNS might provide an avenue for extending stem cell-based disease modeling into an in vivo setting, thereby enabling the visualization of cell-autonomous and non-cell-autonomous pathophenotypes in a largely physiological though xenogeneic whole-brain context. Interesting data along this line have recently been reported in a study focusing on Down syndrome (DS). iPSC-derived NPCs from healthy controls and DS patients were transplanted into the brains of adult mice and traced with two-photon imaging. Neurons from both groups, controls and DS patients, exhibited axonal outgrowth, dendrite pruning and functional connections with host neurons, as indicated by the detection of miniature EPSCs, within an observation period of 4 months. In addition, neuronal bursts of defined spatiotemporal order and recurrent oscillatory behavior resembling neuronal activity in the human developing cortex could be recorded in these grafts. However, in vivo imaging revealed that in comparison to human control neurons, DS-derived neurons demonstrated higher dendritic spine stability marked by a decrease in dendritic spine turnover, which was accompanied by a reduction in network activity and graft-intrinsic oscillation [139]. As neural activity is an important regulator of synaptic plasticity, this study connects alterations in synaptogenesis with aberrant neural activity, which both seem to be at the core of pathogenic processes affecting early neurodevelopment in psychiatric disorders.

As a conceptual alternative to the transplantation of 2D cell culture-derived neural populations, Mansour et al. performed intracerebral transplantation of human brain organoids into the retrosplenial cortex of immunodeficient mice. Interestingly, the authors observed that the engrafted organoids developed long-distance axonal projections to many different brain regions including hippocampus, thalamus and hypothalamus and even underwent synaptic integration. The grafted tissue was also vascularized by host blood vessels, providing promising 
prospects for long-term in vivo studies [140]. In a slightly different scenario, $\mathrm{Xu}$ et al. dissociated control and DS patient-derived organoids into single cells and transplanted them into mouse brains, revealing that DS organoids generated an increased number of functionally active interneurons. Hypothesizing that the overproduction of inhibitory GABAergic neurons might be caused by OLIG2 upregulation, the authors performed shRNAmediated knockdown of OLIG2, which resulted in normalization of interneuron differentiation [141] counteracting $\mathrm{E} / \mathrm{I}$ imbalance.

Considering that dysregulated neuronal subtype generation and maturation might also be a pathomechanism relevant in ASD, these studies collectively suggest that transplantation of PSC-derived patient cells or organoids into an animal brain might be useful to decipher how different cellular processes conspire to elicit ASD phenotypes in a tissue context. Especially aspects such as cell migration, axon outgrowth and synaptic pruning might be more reliably mimicked in a tissue-like context, which provides a more physiological environment with blood vessels, immune cells and a pre-existing neuronal network. As of now, the accurate analysis of neuronal connectivity and network function in these complex systems still remains challenging. However, new functional imaging and reporter tools might open new avenues for assessing electrophysiological activity of both organoids and xenografts in much greater detail.

\section{Available methods for studying neuronal function and circuit formation in stem cell-based models}

The currently available optical tools that enable monitoring of structure and functionality in stem cell-based in vitro models, as well as of grafted cells in vivo, can be divided into three main categories: (1) tools evaluating the structure of neurons and neural circuits, (2) tools reporting neuronal activity and (3) tools that allow the targeted modulation of this activity.

The first tool set mainly comprises virus-based tracer systems for the assessment of morphological and structural properties of neurons and neural circuits in stem cell-derived in vitro models and xenografts, which have already been used in animal models of ASD [20] and might prospectively be implemented in PSC-based disease models as well. In order to express different fluorescent proteins in the human cells of interest, mostly recombinant adeno-associated virus (rAAV)- or lentivirus (LV)-based tracer systems are utilized [142]. Since LVs, unlike rAAVs, integrate into the host cell genome and thus are passed onto the daughter cells after division, they can be efficiently used for transducing proliferative stem cell cultures for subsequent analysis of their neuronal progeny [143], whereas rAAVs are mainly employed to trace post-mitotic neurons [144-146]. Trans-synaptic tracers based on genetically modified recombinant rabies virus (RV) are particularly useful to study the connectivity of human stem cell-derived neurons both in vitro and in vivo after transplantation [145-148]. Using this system, we and others have demonstrated, for instance, that ESC-derived NPCs transplanted into the rodent hippocampus and striatum receive homotopic projections from the host brain $[145,146]$. In a disease context, a RVbased trans-synaptic tracing study has revealed reduced connectivity in iPSC-derived neuronal cultures from patients with $\mathrm{SCZ}$, which could be rescued by treating the SCZ iPSC-derived neurons with the antipsychotic drug loxapine [149]. It is to be expected that these tracing systems might be helpful to more comprehensively assess impaired synaptic connectivity in ASD as well.

The second set of optical tools enables the assessment of functional activity in neurons. The most prominent method for this purpose is calcium imaging, a method that measures variations in the intracellular calcium concentration as a proxy for neuronal activity, and calcium sensors such as Fluo-4AM were already successfully employed to study iPSC-based ASD models in 2D cultures [53] and 3D organoids [135]. These studies revealed a decreased frequency in calcium oscillations in RTS-derived neurons [53], whilst an increase in the overall intracellular calcium concentration was observed in neurons derived from patients with Timothy syndrome [135]. Genetically encoded calcium-indicators provide another route to assess functionality of grafted neurons. This was impressively illustrated by a recent study from Vanderhaeghen's group, which demonstrated that grafted human ESC-derived cortical neurons become functional and undergo network integration in the mouse visual cortex [148]. Healthy iPSC-derived brain organoids grafted into the mouse brain are functionally active, too, as indicated by the occurrence of calcium oscillations in excitatory neurons within the transplanted organoid [140]. Voltage sensors responsive to changes in the cell membrane potential have been recombinantly expressed in healthy iPSC-derived neurons, too, and used to detect the occurrence of homeostatic plasticity of cell-intrinsic excitability-a very subtle form of synaptic plasticity, which had so far not been observed in human iPSCderived neurons - that previously required laborious and time-consuming patch clamping [150]. Consequently, the use of such indicators might become especially attractive for potential future applications taking diseased iPSCderived cells into a whole-brain context.

Lastly, the third set of optical tools comprising optically activated proteins, such as channelrhodopsin-II and halorhodopsins, allows to either excite (depolarize) or inhibit (hyperpolarize) neurons using blue or yellow 
light, respectively. These methods have been successfully used to study the effects of membrane potential changes in neurons evolving from mouse [151] and human ESCs in vitro [140], as well as to control the activity of engrafted neurons [140,143, 152]. In the context of a Parkinson's disease animal model, for instance, transplantation of ESC-derived dopaminergic neurons ameliorates functional deficits, and light-induced suspension of graft activity abolishes this beneficial effect on amphetamineinduced rotation behavior [153]. Although these techniques have not yet been exploited in the context of ASD, they could potentially be used to selectively excite or inhibit neuronal subpopulations derived from patient PSCs, e.g., in order to decipher the relative contribution of neuronal subtypes to network activity and E/I (im) balance.

With the increasing move towards tissue-based models such as organoids and transplants, imaging technologies, too, need to be tailored towards more elaborate 3D analyses. Conventional sectioning and post-hoc immunohistochemistry of organoids or brains results in comparably limited 3D representation. Here, whole mount imaging techniques such as tissue clearing in conjunction with high-resolution 3D light sheet fluorescence imaging provide fast and reliable alternatives. This approach was, for instance, successfully used for assessing axonal projections of small molecule-induced cortical neurons [154] and RVbased trans-synaptic tracing of host-graft innervation [146]. Notably, light sheet fluorescence imaging of cleared tissue can also be combined with novel tissue expansion protocols allowing the depiction of neuronal circuits and synaptic connections in super-resolution [155].

In addition to optical tools and classical electrophysiological methods such as patch clamping, multi-electrode arrays (MEAs) have been used successfully to assess neuronal activity of stem cell-based systems. This technology enables functional recordings from PSC-derived neurons on a population level, including the assessment of network-related parameters such as network burst activity and synchrony. Notably, this technology is sensitive enough to detect subtle alterations in network activity such as, for instance, decreased synchronized bursting after inactivation of L-type calcium channels [156], which are implicated in neuropsychiatric disorders such as ASD [65]. The parameters assessed by MEAs are likely to be affected in the context of an E/I imbalance or other functional alterations discussed in the context of ASD, as was already demonstrated in an iPSC-based 2D model of idiopathic ASD [88]. While Trujillo et al. recently reported that MEAs can be used to detect oscillatory waves and neuronal network activity in long-term cultured healthy brain organoids [157], future studies might also exploit this technology for organoid-based disease modeling.

\section{Next generation tools for assessing neuronal connectivity and function in vivo}

During the last couple of years, a vast array of additional high-end tools for assessing neuronal function and connectivity have been established in rodent systems. Translated to human stem cell-derived neurons, these tools could provide unprecedented insights into functional alterations associated with ASD and other neuropsychiatric diseases. As these read-out systems are streamlined towards application in the rodent brain, they might be particularly useful for studying altered connectivity and function of human neurons grafted into a rodent brain (Box 1).

Selected examples of novel tools originally developed for assessing connectivity and neuronal function in the rodent brain might lend themselves to the analysis of patient iPSC-derived neurons in 3D cultures and xenografts. Tools developed to study anatomical features include the Brainbow technology [158] and the novel Tetbow system recently employed in the context of rAAV-based labeling studies [159]. Both approaches enable labeling of multiple cells with individual colors and can thus provide a detailed unambiguous insight into neurite projection patterns.

Besides the mere detection and visualization of synaptically connected cells using well-established recombinant RV-based systems, the TRIO technology could serve as a valuable tool for assessing graft integration into the host circuitry. TRIO uses three different virus types to determine the input and output connectivity of diverse neuronal populations; the most recent cTRIO variant even confers cell-type specificity [160].

While these methods label synaptically connected neurons as such, the mGRASP system can be used to visualize individual synapses engaged in neuronal communication. The technology is based on functional complementation between two non-fluorescent GFP fragments across tightly juxtapositioned pre- and postsynaptic membranes [161] and can even be used in combination with optogenetic tools [162].

Novel techniques such as MAPseq, SYNseq and BARseq combine RNA barcoding and projection mapping, thereby enabling transcriptome analyses in connected cells [163-165] - certainly an interesting perspective for the study of graft-host interactions.

Another toolset comprises methods for the evaluation of a neurons' activity state as well as detailed information on synaptic neurotransmitter release. Here, deeper insight into the actual function of stem cell-derived neurons could be achieved with advanced optical activity sensors and modulators. Currently applied conventional calcium sensors visualize neuronal activity in real time, posing limitations for post hoc analysis though. Since the 


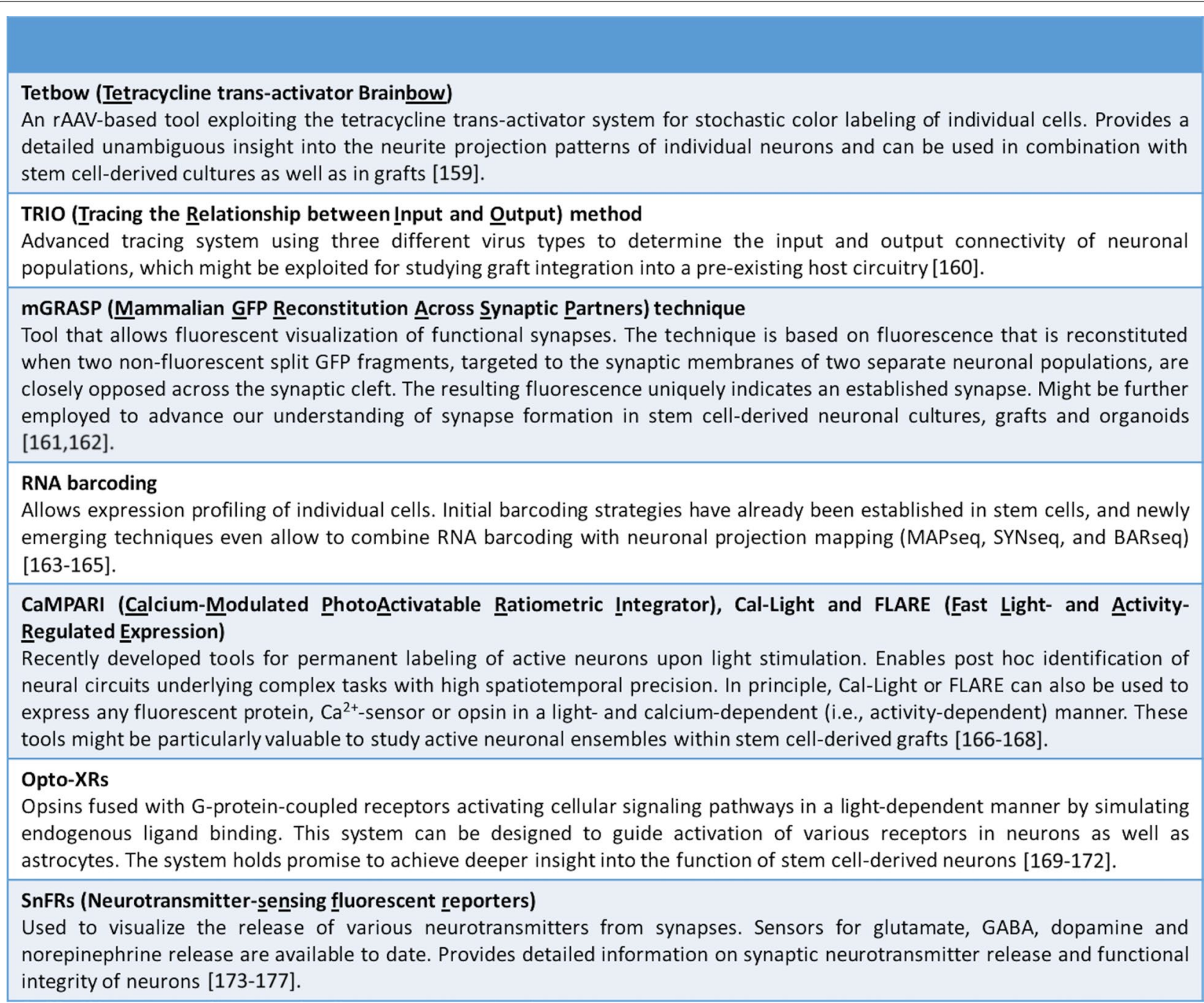

Box 1 Next generation tools for assessing neuronal connectivity and function in vivo

fluorescence signal is lost after fixation, it is impossible to retrospectively determine, for instance, the cellular identity of the cells that have been engaged in neuronal activity within a piece of brain tissue. The recently developed activity sensor CaMPARI overcomes this limitation via a permanent green-to-red fluorescence conversion upon blue light stimulation [166]. As a result, CaMPARI allows for post hoc identification of active neurons and might thus be used in the context of stem cell grafts to permanently and selectively label active neurons at a specific point of time, which would allow an accurate evaluation of the number and type of neurons engaged in activity within a xenograft. Optical switch systems such as CalLight or FLARE operate along the same line and can, in principle, be used to express any fluorescent protein, calcium sensor or opsin in a light- and calcium-dependent manner exclusively in active neurons. This is not only attractive for studying neuronal dynamics within human transplants, but also for modulating the activity of grafted cells $[167,168]$.

Other variants for advanced optical activity modulation include Opto-XRs, which are opsins fused with G-protein coupled receptors, thus activating cellular signaling pathways in a light-dependent manner by simulating endogenous ligand binding. In vivo, Opto-XRs have been successfully used to study the role of different receptors in regulating complex behavior [169-172].

Finally, and a level deeper in complexity, neurotransmitter-sensing fluorescent reporters (SnFRs) even enable visualization of transmitter release from neuronal synapses and astrocytes; a number of SnFRs detecting synaptic glutamate, GABA, dopamine and norepinephrine release have already been established [173-177]. 
Despite the reasonable excitement accompanying these technological advances, implementation of such complex read-outs for studying disease-related alterations in human 3D cultures and xenografted human neurons will be challenging. Donor cell preparations will have to be highly defined and homogenous to minimize intergraft variability. Increased heterogeneity, as currently experienced, for example, in many organoid preparations and grafts, could result in highly variable and hardly interpretable connectivity patterns. For example, surgical variability in graft placement, differing degrees of cell survival, heterogeneity with respect to in situ cell maturation and immunological factors could significantly interfere with the functional analysis of human neurons in 3D cultures and xenografts.

\section{Stem cell-based models for neuropsychiatric drug development}

Besides increasing our understanding of the pathophysiological mechanisms underlying neuropsychiatric diseases, PSC-based models represent valuable platforms for the identification and validation of candidate pharmacological compounds for the treatment of these disorders. Kumari et al. [178] and Kaufmann et al. [179], for instance, screened thousands of compounds for their ability to increase FMR1 expression. Although the authors only identified a small number of compounds exhibiting modest efficacy, including an HDAC inhibitor [179], these studies illustrate the feasibility of using PSC-derived disease models for drug screening. More recently, Vershkov et al. used FXS iPSCs to test a library of 140 compounds for their ability to rescue the expression of FMR1. A combination of the DNA methyltransferase inhibitor 5-azadC and the SAH hydrolase and histone methylation inhibitor DZNep was most effective in iPSCs, whereas a sustained increase in FMR1 expression in FXS iPSC-derived NPCs was even observed after treatment with 5-azadC alone. Importantly, such treatments were also effective in vivo, since treatment with 5-azadC and DZNep or 5-azadC alone after transplantation of FXS iPSCs or FXS iPSC-derived NPCs into mouse brains, respectively, resulted in a sustained increase in FMR1 expression [180]. Along a similar line, Darville et al. screened more than 200 compounds for their capability to increase SHANK3 expression in iPSCderived neurons from patients with SHANK3 haploinsufficiency and identified lithium and VPA as most effective candidates in this regard. These two compounds even increased the number of SHANK3-containing synapses and successfully reverted the decreased intensities and frequencies of spontaneous calcium oscillations associated with SHANK3 haploinsufficiency. As a proof-ofconcept for the validity of their screening results, the authors administered lithium to an ASD patient, whose iPSC-derived neurons exhibited increased SHANK3 expression upon lithium exposure in vitro. After 1 year of lithium administration, this patient indeed showed improved social behavior and cognitive performance [181]. These preliminary findings point to interesting prospects of the iPSC technology for personalized medicine, as they indicate that patient-specific iPSC-derived neurons could, in principle, be employed as a platform to screen for effective compounds in an individualized manner.

\section{Future perspectives and concluding remarks}

Although highly defined PSC-derived neuronal cultures facilitate the study of cell type-specific alterations relevant for psychiatric disorders, they do not represent physiological neuronal circuitries, which are composed of a variety of different neuronal as well as glial cell types. On the other hand, reductionist approaches may come with their own advantages. For example, for assessing E/I balance-a phenomenon discussed as relevant for a number of psychiatric diseases-highly defined populations of excitatory and inhibitory neurons generated by forward programming could be very helpful. Stoichiometrically mixed and equipped with distinct fluorescent reporter genes, such co-culture systems could reveal how each individual cell type contributes to altered network formation and activity in ASD. More complex disease models based on the co-culture of neuronal and non-neuronal cells such as astrocytes, oligodendrocytes and microglia might be informative for investigating non-cell-autonomous effects relevant for disease development. Whilst such advanced 2D culture systems might be well suited for high-throughput genetic and compound screens, 3D settings such as organoids or neurotransplantation could provide a tissue-like 3D environment and thus increase the authenticity of human neuronal model systems. In contrast to classic animal models, xenografts provide the possibility to investigate human-specific gene variants in human cells in vivo. This can provide an advantage over mouse models because many disease-related genes do not have mouse orthologs and human cells might respond different to pathogenic stimuli than mouse cells [182]. Prospectively, xenograft approaches could also open an avenue to assess the effect of compounds on disease-relevant human cells in a living mammalian brain.

In conclusion, PSC-based 2D and 3D models represent powerful platforms to (1) investigate the pathophysiological mechanisms underlying genetically defined and potentially idiopathic forms of ASD, (2) test how environmental factors such as prenatal exposure to certain chemicals and drugs predisposes to ASD and (3) identify new treatment strategies for this neuropsychiatric 
disease. While there is a need to further improve the generation of highly standardized cell systems, the combination of iPSC technology, genome editing and novel functional read-out systems has the makings to transform our capabilities for studying the pathogenesis and treatment of neuronal dysfunction in ASD and other neuropsychiatric disorders.

\section{Abbreviations}

AAVS1: Adeno-associated virus site 1; AFF2: AF4/FMR2 family member 2; AKT (aka PKB): Protein kinase B; AMPAR: a-Amino-3-hydroxy-5-methyl-4isoxazolepropionic acid receptor; ANOS1: Anosmin 1; ASCL1: Achaete-scute family bHLH transcription factor 1; ASD: Autism spectrum disorder; ASTN2: Astrotactin 2; ATRX: ATRX chromatin remodeler; 5-azadC: 5'-Aza-2'deoxycytidine; BK channels: Big potassium channels; BRN2 (aka POU3F2): Brain-2; CACNA1C: Calcium voltage-gated channel subunit alpha 1 C; CDKL5: Cyclin-dependent kinase-like 5; CHD8: Chromodomain helicase DNA-binding protein 8; CLCN3: Chloride voltage-gated channel 3; CNS: Central nervous system; CNV: Copy number variation; CREB: CAMP (Cyclic adenosine monophosphate) response element-binding protein; CRISPR/Cas9: Clustered regularly interspaced short palindromic repeats (CRISPR)-associated protein 9; CTIP2: COUP (chicken ovalbumin upstream promoter)-transcription factor-interacting protein 2; DCC: DCC netrin 1 receptor; DISC1: Disrupted in schizophrenia 1; DLGAP2: DLG-associated protein 2; DLX: Distal-less homeobox; DNT: Developmental neurotoxicity; DS: Down syndrome; DZNep: 3-Deazaneplanocin A; EHMT1: Euchromatin histone lysine methyltransferase 1; E/l: Excitation-inhibition; EPSC: Excitatory postsynaptic current; ESC: Embryonic stem cell; FMR1: FMRP translational regulator 1; FMRP: Fragile X mental retardation 1 protein; FOXG1: Forkhead box G1; FXS: Fragile X syndrome; FURIN: Coding for furin, paired basic amino acid cleaving enzyme; GABA: Gamma aminobutyric acid; GRIA: Glutamate ionotropic receptor AMPA-type subunit; GRIN: Glutamate ionotropic receptor NMDA-type subunit; GRM: Glutamate metabotropic receptor; IGF1: Insulin-like growth factor 1; IL-6: Interleukin 6; iPSC: Induced pluripotent stem cell; KCC2 (aka SLC12A5): Potassium chloride transporter member 5; KCNQ2: Potassium voltage-gated channel subfamily Q member; KO: Knockout; LV: Lentivirus; MEAs: Multi-electrode arrays; MECP2: MethylCpG-binding protein 2; MEKJERK: Mitogen-activated protein kinase (MAPK)/ extracellular signal-regulated kinase (Erk) kinase; MFN1: Mitofusin 1; mGluR5: Metabotropic glutamate receptor 5; MRI: Magnetic resonance imaging; mTOR: Mechanistic target of rapamycin; NCAM: Neural cell adhesion molecule; NDE1: NudE neurodevelopment protein 1; NDEL1: NudE neurodevelopment protein 1-like 1; NGN2: Neurogenin 2; NLGN3/4: Neuroligin 3/4; NMDA: N-Methyl-D-aspartic acid; NPC: Neural progenitor cell; NRXN1: Neurexin 1; OTX2: Orthodenticle homeobox 2; PAX6: Paired box 6; PI3K: Phosphoinositide 3 kinase; PSC: Pluripotent stem cell; PSD95: Postsynaptic density protein 95; PTCHD1-AS: Patched domain-containing protein 1-antisense RNA; rAAV: Recombinant adeno-associated virus; RAB39B: Ras-related protein Rab-39B; REST: RE1 silencing transcription factor; $\mathrm{ROBO}$ : Roundabout guidance receptor 3; RTS: Rett syndrome; RV: Rabies virus; SAH: S-Adenosylhomocysteine; SCN2A: Sodium voltage-gated channel alpha subunit 2; SCZ: Schizophrenia; SHANK2/3: SH3 and multiple ankyrin repeat domains 2/3; SHH: Sonic hedgehog signaling molecule; SLIT1: Slit guidance ligand 1; SMAD: Small mothers against decapentaplegic; SNAP91: Synaptosome-associated protein 91; SNP: Single nucleotide polymorphism; SOX1: SRY (sex-determining region Y)-box transcription factor 1; TBR1: T-box brain transcription factor 1; TENM1: Teneurin transmembrane protein 1; TRPC6: Short transient receptor potential channel 6; TSNARE1:T-SNARE domain-containing 1; UBE3A: Ubiquitin protein ligase E3A; VGLUT: Vesicular glutamate transporter; VPA: Valporic acid; WNT: Wingless and int-1.

\section{Authors' contributions}

All authors contributed to writing the manuscript. AC, LF and OB conducted the final assembly. All authors read and approved the final manuscript.

\section{Funding}

Open Access funding enabled and organized by Projekt DEAL. This work was supported by COSYN (Comorbidity and Synapse Biology in Clinically
Overlapping Psychiatric Disorders, Horizon 2020 Program of the European Union under RIA Grant Agreement 667301); TREAT-SNGAP (the ERA-Net Neuron program, Grant 01EW1812A—Synaptic Dysfunction in Intellectual Disability Caused by SYNGAP1-TREAT-SNGAP); SCFIII (the European Regional Development Fund (Stem Cell Factory III; Grant EFRE-0800978); and BfArM (the Federal Institute for Drugs and Medical Devices, Grant V-17665/68502/2017-2021).

\section{Availability of data and materials \\ Not applicable.}

Ethics approval and consent to participate

Not applicable.

\section{Consent for publication}

Not applicable.

\section{Competing interests}

Not applicable.

\section{Author details}

${ }^{1}$ Institute of Reconstructive Neurobiology, University of Bonn Medical Faculty \& University Hospital Bonn, Venusberg-Campus 1, Building 76, 53127 Bonn, Germany. ${ }^{2}$ Life \& Brain GmbH, Platform Cellomics, Venusberg-Campus 1, Building 76, 53127 Bonn, Germany. ${ }^{3}$ Institute of Experimental Epileptology and Cognition Research, University of Bonn Medical Faculty \& University Hospital Bonn, Venusberg-Campus 1, Building 76, 53127 Bonn, Germany. ${ }^{4}$ Cell Programming Core Facility, University of Bonn Medical Faculty, Bonn, Germany.

Received: 5 June 2020 Accepted: 22 September 2020

Published online: 11 December 2020

\section{References}

1. American Psychiatric Association. Diagnostic and statistical manual of mental disorders. 5th ed. Washington: American Psychiatric Association; 2013.

2. Miles JH. Autism spectrum disorders—a genetics review. Genet Med. 2011;13:278-94.

3. Sztainberg Y, Zoghbi HY. Lessons learned from studying syndromic autism spectrum disorders. Nat Neurosci. 2016;19:1408-17.

4. Caglayan AO. Genetic causes of syndromic and non-syndromic autism. Dev Med Child Neurol. 2010;52:130-8.

5. Modabbernia A, Velthorst E, Reichenberg A. Environmental risk factors for autism: an evidence-based review of systematic reviews and metaanalyses. Mol Autism. 2017;8:13.

6. SPARK Consortium. SPARK: a US Cohort of 50,000 families to accelerate Autism Research. Neuron. 2018;97:488-93.

7. Schaefer GB, Starr L, Pickering D, Skar G, Dehaai K, Sanger WG. Array comparative genomic hybridization findings in a cohort referred for an autism evaluation. J Child Neurol. 2010;25:1498-503.

8. Reuter MS, Tawamie H, Buchert R, Hosny Gebril O, Froukh T, Thiel C, et al. Diagnostic yield and novel candidate genes by exome sequencing in 152 consanguineous families with neurodevelopmental disorders. JAMA Psychiatry. 2017;74:293-9.

9. Verkerk AJ, Pieretti M, Sutcliffe JS, Fu YH, Kuhl DP, Pizzuti A, et al. Identification of a gene (FMR-1) containing a CGG repeat coincident with a breakpoint cluster region exhibiting length variation in fragile $\mathrm{X}$ syndrome. Cell. 1991;65:905-14.

10. Amir RE, Van den Veyver IB, Wan M, Tran CQ, Francke U, Zoghbi HY. Rett syndrome is caused by mutations in X-linked MECP2, encoding methylCpG-binding protein 2. Nat Genet. 1999;23:185-8.

11. Telias M, Segal M, Ben-Yosef D. Neural differentiation of fragile $X$ human embryonic stem cells reveals abnormal patterns of development despite successful neurogenesis. Dev Biol. 2013;374:32-45.

12. Nomura T, Musial TF, Marshall JJ, Zhu Y, Remmers $\mathrm{CL}$, Xu J, et al. Delayed maturation of fast-spiking interneurons is rectified by activation of the TrkB Receptor in the mouse model of fragile X syndrome. J Neurosci. 2017;37:11298-310. 
13. Curia G, Papouin T, Séguéla P, Avoli M. Downregulation of tonic GABAergic inhibition in a mouse model of fragile X syndrome. Cereb Cortex. 2009;19:1515-20.

14. Michalon A, Sidorov M, Ballard TM, Ozmen L, Spooren W, Wettstein JG, et al. Chronic pharmacological mGlu5 inhibition corrects fragile X in adult mice. Neuron. 2012;74:49-56.

15. Scharkowski F, Frotscher M, Lutz D, Korte M, Michaelsen-Preusse K. Altered connectivity and synapse maturation of the hippocampal mossy fiber pathway in a mouse model of the fragile $X$ syndrome. Cereb Cortex. 2018;28:852-67.

16. Patel AB, Loerwald KW, Huber KM, Gibson JR. Postsynaptic FMRP promotes the pruning of cell-to-cell connections among pyramidal neurons in the L5A neocortical network. J Neurosci. 2014;34:3413-8.

17. Tao J, Wu H, Coronado AA, de Laittre E, Osterweil EK, Zhang Y, et al. Negative allosteric modulation of mGluR5 partially corrects pathophysiology in a mouse model of Rett syndrome. J Neurosci. 2016;36:11946-58.

18. Patrizi A, Picard N, Simon AJ, Gunner G, Centofante E, Andrews NA, et al. Chronic administration of the $\mathrm{N}$-methyl-D-aspartate receptor antagonist ketamine improves Rett syndrome phenotype. Biol Psychiatry. 2016;79:755-64.

19. Cobolli Gigli C, Scaramuzza L, De Simone M, Rossi RL, Pozzi D, Pagani $\mathrm{M}$, et al. Lack of methyl-CpG binding protein 2 (MeCP2) affects cell fate refinement during embryonic cortical development. Cereb Cortex. 2018:28:1846-56.

20. Sun Y, Gao Y, Tidei JJ, Shen M, Hoang JT, Wagner DF, et al. Loss of MeCP2 in immature neurons leads to impaired network integration. Hum Mol Genet. 2019;28:245-57.

21. Salpietro V, Dixon CL, Guo H, Bello OD, Vandrovcova J, Efthymiou S, et al. AMPA receptor GluA2 subunit defects are a cause of neurodevelopmental disorders. Nat Commun. 2019;10:3094.

22. Soto D, Altafaj $X$, Sindreu C, Bayés A. Glutamate receptor mutations in psychiatric and neurodevelopmental disorders. Commun Integr Biol. 2014;7:e27887.

23. Courchesne E, Karns CM, Davis HR, Ziccardi R, Carper RA, Tigue ZD, et al. Unusual brain growth patterns in early life in patients with autistic disorder: an MRI study. Neurology. 2001;57:245-54.

24. Courchesne E, Campbell K, Solso S. Brain growth across the life span in autism: age-specific changes in anatomical pathology. Brain Res. 2011;1380:138-45.

25. lidaka T, Kogata T, Mano Y, Komeda H. Thalamocortical hyperconnectivity and amygdala-cortical hypoconnectivity in male patients with autism spectrum disorder. Front Psychiatry. 2019;10:252.

26. Hutsler JJ, Zhang H. Increased dendritic spine densities on cortical projection neurons in autism spectrum disorders. Brain Res. 2010;1309:83-94.

27. Roden WH, Peugh LD, Jansen LA. Altered GABA(A) receptor subunit expression and pharmacology in human Angelman syndrome cortex. Neurosci Lett. 2010;483:167-72.

28. Morin-Parent F, Champigny C, Lacroix A, Corbin F, Lepage J-F. Hyperexcitability and impaired intracortical inhibition in patients with fragile-X syndrome. Transl Psychiatry. 2019;9:312.

29. Zhou Y, Sharma J, Ke Q, Landman R, Yuan J, Chen H, et al. Atypical behaviour and connectivity in SHANK3-mutant macaques. Nature. 2019;570:326-31.

30. Takahashi K, Tanabe K, Ohnuki M, Narita M, Ichisaka T, Tomoda K, et al. Induction of pluripotent stem cells from adult human fibroblasts by defined factors. Cell. 2007;131:861-72.

31. Takahashi K, Yamanaka S. Induction of pluripotent stem cells from mouse embryonic and adult fibroblast cultures by defined factors. Cell. 2006;126:663-76.

32. Kim J-H, Kurtz A, Yuan B-Z, Zeng F, Lomax G, Loring JF, et al. Report of the international stem cell banking initiative workshop activity: current hurdles and progress in seed-stock banking of human pluripotent stem cells. Stem Cells Transl Med. 2017;6:1956-62.

33. O'Shea O, Steeg R, Chapman C, Mackintosh P, Stacey GN. Development and implementation of large-scale quality control for the European bank for induced Pluripotent Stem Cells. Stem Cell Res. 2020;45:101773.

34. Lu C, Shi X, Allen A, Baez-Nieto D, Nikish A, Sanjana NE, et al. Overexpression of NEUROG2 and NEUROG1 in human embryonic stem cells produces a network of excitatory and inhibitory neurons. FASEB $\mathrm{J}$. 2019:33:5287-99.
35. Chambers SM, Fasano CA, Papapetrou EP, Tomishima M, Sadelain M, Studer L. Highly efficient neural conversion of human ES and iPS cells by dual inhibition of SMAD signaling. Nat Biotechnol. 2009;27:275-80

36. Shi Y, Kirwan P, Smith J, Robinson HPC, Livesey FJ. Human cerebral cortex development from pluripotent stem cells to functional excitatory synapses. Nat Neurosci. 2012;15:477-486.S1.

37. Rehbach K, Kesavan J, Hauser S, Ritzenhofen S, Jungverdorben J, Schüle $R$, et al. Multiparametric rapid screening of neuronal process pathology for drug target identification in HSP patient-specific neurons. Sci Rep. 2019;9:9615.

38. Rhee HJ, Shaib AH, Rehbach K, Lee C, Seif P, Thomas C, et al. An autaptic culture system for standardized analyses of iPSC-derived human neurons. Cell Rep. 2019;27:2212-2228.e7.

39. Maroof AM, Keros S, Tyson JA, Ying S-W, Ganat YM, Merkle FT, et al. Directed differentiation and functional maturation of cortical interneurons from human embryonic stem cells. Cell Stem Cell. 2013;12:559-72.

40. Nicholas CR, Chen J, Tang Y, Southwell DG, Chalmers N, Vogt D, et al. Functional maturation of hPSC-derived forebrain interneurons requires an extended timeline and mimics human neural development. Cell Stem Cell. 2013;12:573-86.

41. Zhang Y, Pak C, Han Y, Ahlenius H, Zhang Z, Chanda S, et al. Rapid single-step induction of functional neurons from human pluripotent stem cells. Neuron. 2013;78:785-98.

42. Yang N, Chanda S, Marro S, Ng Y-H, Janas JA, Haag D, et al. Generation of pure GABAergic neurons by transcription factor programming. Nat Methods. 2017;14:621-8.

43. Meijer M, Rehbach K, Brunner JW, Classen JA, Lammertse HCA, van Linge LA, et al. A single-cell model for synaptic transmission and plasticity in human iPSC-derived neurons. Cell Rep. 2019;27(2199-2211):e6.

44. Germain P-L, Testa G. Taming human genetic variability: transcriptomic meta-analysis guides the experimental design and interpretation of iPSC-based disease modeling. Stem Cell Rep. 2017:8:1784-96.

45. Kirov G, Rees E, Walters JTR, Escott-Price V, Georgieva L, Richards AL, et al. The penetrance of copy number variations for schizophrenia and developmental delay. Biol Psychiatry. 2014;75:378-85.

46. Wayman GA, Impey S, Marks D, Saneyoshi T, Grant WF, Derkach V, et al. Activity-dependent dendritic arborization mediated by CaM-kinase I activation and enhanced CREB-dependent transcription of Wnt-2. Neuron. 2006;50:897-909

47. Klein ME, Lioy DT, Ma L, Impey S, Mandel G, Goodman RH. Homeostatic regulation of MeCP2 expression by a CREB-induced microRNA. Nat Neurosci. 2007;10:1513-4.

48. Bu Q, Wang A, Hamzah H, Waldman A, Jiang K, Dong Q, et al. CREB signaling is involved in Rett syndrome pathogenesis. J Neurosci. 2017;37:3671-85.

49. Deneault E, White SH, Rodrigues DC, Ross PJ, Faheem M, Zaslavsky K, et al. Complete disruption of autism-susceptibility genes by gene editing predominantly reduces functional connectivity of isogenic human neurons. Stem Cell Rep. 2018;11:1211-25.

50. Schrode N, Ho S-M, Yamamuro K, Dobbyn A, Huckins L, Matos MR, et al. Synergistic effects of common schizophrenia risk variants. Nat Genet. 2019;51:1475-85.

51. Achuta VS, Möykkynen T, Peteri U-K, Turconi G, Rivera C, Keinänen $\mathrm{K}$, et al. Functional changes of AMPA responses in human induced pluripotent stem cell-derived neural progenitors in fragile X syndrome. Sci Signal. 2018;11:eaan8784

52. Halevy T, Czech C, Benvenisty N. Molecular mechanisms regulating the defects in fragile $X$ syndrome neurons derived from human pluripotent stem cells. Stem Cell Rep. 2015;4:37-46.

53. Marchetto MCN, Carromeu C, Acab A, Yu D, Yeo GW, MuY, et al. A mode for neural development and treatment of Rett syndrome using human induced pluripotent stem cells. Cell. 2010;143:527-39.

54. Tang X, Kim J, Zhou L, Wengert E, Zhang L, Wu Z, et al. KCC2 rescues functional deficits in human neurons derived from patients with Rett syndrome. Proc Natl Acad Sci U S A. 2016;113:751-6.

55. Keeling KM, Bedwell DM. Clinically relevant aminoglycosides can suppress disease-associated premature stop mutations in the IDUA and P53 CDNAs in a mammalian translation system. J Mol Med (Berl). 2002;80:367-76. 
56. Tropea D, Giacometti E, Wilson NR, Beard C, McCurry C, Fu DD, et al. Partial reversal of Rett syndrome-like symptoms in MeCP2 mutant mice. Proc Natl Acad Sci U S A. 2009;106:2029-34.

57. Djuric U, Cheung AYL, Zhang W, Mok RS, Lai W, Piekna A, et al. MECPZe1 isoform mutation affects the form and function of neurons derived from Rett syndrome patient iPS cells. Neurobiol Dis. 2015;76:37-45.

58. Nageshappa S, Carromeu C, Trujillo CA, Mesci P, Espuny-Camacho I, Pasciuto $E$, et al. Altered neuronal network and rescue in a human MECP2 duplication model. Mol Psychiatry. 2016;21:178-88.

59. Srivastava S, Desai S, Cohen J, Smith-Hicks C, Barañano K, Fatemi A, et al. Monogenic disorders that mimic the phenotype of Rett syndrome. Neurogenetics. 2018;19:41-7.

60. Ricciardi S, Ungaro F, Hambrock M, Rademacher N, Stefanelli G, Brambilla $D$, et al. CDKL5 ensures excitatory synapse stability by reinforcing NGL-1-PSD95 interaction in the postsynaptic compartment and is impaired in patient iPSC-derived neurons. Nat Cell Biol. 2012;14:911-23.

61. Chamberlain SJ, Lalande M. Angelman syndrome, a genomic imprinting disorder of the brain. J Neurosci. 2010;30:9958-63.

62. Fink JJ, Robinson TM, Germain ND, Sirois CL, Bolduc KA, Ward AJ, et al. Disrupted neuronal maturation in Angelman syndrome-derived induced pluripotent stem cells. Nat Commun. 2017;8:15038.

63. Fink JJ, Schreiner JD, Bloom JE, Baker DS, Robinson TM, Lieberman R, et al. Hyperexcitable phenotypes in iPSC-derived neurons from patients with 15q11-q13 duplication syndrome, a genetic form of autism. bioRxiv. 2018;286336.

64. Sun AX, Yuan Q, Fukuda M, Yu W, Yan H, Lim GGY, et al. Potassium channel dysfunction in human neuronal models of Angelman syndrome. Science. 2019;366:1486-92.

65. Paşca SP, Portmann T, Voineagu I, Yazawa M, Shcheglovitov A, Paşca AM, et al. Using iPSC-derived neurons to uncover cellular phenotypes associated with Timothy syndrome. Nat Med. 2011;17:1657-62.

66. Monteiro P, Feng G. SHANK proteins: roles at the synapse and in autism spectrum disorder. Nat Rev Neurosci. 2017;18:147-57.

67. Boeckers TM, Bockmann J, Kreutz MR, Gundelfinger ED. ProSAP/Shank proteins - a family of higher order organizing molecules of the postsynaptic density with an emerging role in human neurological disease. J Neurochem. 2002;81:903-10.

68. Huang G, Chen S, Chen X, Zheng J, Xu Z, Doostparast Torshizi A, et al. Uncovering the functional link between SHANK3 deletions and deficiency in neurodevelopment using iPSC-derived human neurons. Front Neuroanat. 2019;13:23.

69 Yi F, Danko T, Botelho SC, Patzke C, Pak C, Wernig M, et al. Autism-associated SHANK3 haploinsufficiency causes Ih channelopathy in human neurons. Science. 2016;352:aaf669.

70. Gouder L, Vitrac A, Goubran-Botros H, Danckaert A, Tinevez J-Y, AndréLeroux G, et al. Altered spinogenesis in iPSC-derived cortical neurons from patients with autism carrying de novo SHANK3 mutations. Sci Rep. 2019;9:94.

71. Kathuria A, Nowosiad P, Jagasia R, Aigner S, Taylor RD, Andreae LC, et al. Stem cell-derived neurons from autistic individuals with SHANK3 mutation show morphogenetic abnormalities during early development. Mol Psychiatry. 2018;23:735-46.

72. Paoletti P, Bellone C, Zhou Q. NMDA receptor subunit diversity: impact on receptor properties, synaptic plasticity and disease. Nat Rev Neurosci. 2013;14:383-400.

73. Shcheglovitov A, Shcheglovitova O, Yazawa M, Portmann T, Shu R, Sebastiano $V$, et al. SHANK3 and IGF1 restore synaptic deficits in neurons from 22q13 deletion syndrome patients. Nature. 2013;503:267-71.

74. Frega M, Linda K, Keller JM, Gümüş-Akay G, Mossink B, van Rhijn J-R, et al. Neuronal network dysfunction in a model for Kleefstra syndrome mediated by enhanced NMDAR signaling. Nat Commun. 2019;10:4928.

75. Sala C, Vicidomini C, Bigi I, Mossa A, Verpelli C. Shank synaptic scaffold proteins: keys to understanding the pathogenesis of autism and other synaptic disorders. J Neurochem. 2015;135:849-58.

76. Zaslavsky K, Zhang W-B, McCready FP, Rodrigues DC, Deneault E, Loo C, et al. SHANK2 mutations associated with autism spectrum disorder cause hyperconnectivity of human neurons. Nat Neurosci. 2019;22:556-64.

77. Marro SG, Chanda S, Yang N, Janas JA, Valperga G, Trotter J, et al. Neuroligin-4 regulates excitatory synaptic transmission in human neurons. Neuron. 2019;103(617-626):e6.
78. Avazzadeh S, McDonagh K, Reilly J, Wang Y, Boomkamp SD, Mclnerney $\checkmark$, et al. Increased $\mathrm{Ca}^{2+}$ signaling in $\mathrm{NRXN1a}^{+/-}$neurons derived from ASD induced pluripotent stem cells. Mol Autism. 2019;10:52.

79. Lam M, Moslem M, Bryois J, Pronk RJ, Uhlin E, Ellström ID, et al. Single cell analysis of autism patient with bi-allelic NRXN1-alpha deletion reveals skewed fate choice in neural progenitors and impaired neuronal functionality. Exp Cell Res. 2019;383:111469.

80. Pak C, Danko T, Zhang Y, Aoto J, Anderson G, Maxeiner S, et al. Human neuropsychiatric disease modeling using conditional deletion reveals synaptic transmission defects caused by heterozygous mutations in NRXN1. Cell Stem Cell. 2015;17:316-28.

81. Flaherty E, Zhu S, Barretto N, Cheng E, Deans PJM, Fernando MB, et al. Neuronal impact of patient-specific aberrant NRXN1a splicing. Nat Genet. 2019:51:1679-90.

82. Green Snyder L, D'Angelo D, Chen Q, Bernier R, Goin-Kochel RP, Wallace AS, et al. Autism spectrum disorder, developmental and psychiatric features in 16p11.2 duplication. J Autism Dev Disord. 2016;46:2734-48.

83. Deshpande A, Yadav S, Dao DQ, Wu Z-Y, Hokanson KC, Cahill MK, et al. Cellular phenotypes in human iPSC-derived neurons from a genetic model of autism spectrum disorder. Cell Rep. 2017;21:2678-87.

84. Griesi-Oliveira K, Acab A, Gupta AR, Sunaga DY, Chailangkarn T, Nicol X, et al. Modeling non-syndromic autism and the impact of TRPC6 disruption in human neurons. Mol Psychiatry. 2015;20:1350-65.

85. Ross PJ, Zhang W-B, Mok RSF, Zaslavsky K, Deneault E, D'Abate L, et al. Synaptic dysfunction in human neurons with autism-associated deletions in PTCHD1-AS. Biol Psychiatry. 2020;87:139-49.

86. Liu X, Campanac E, Cheung H-H, Ziats MN, Canterel-Thouennon L, Raygada M, et al. Idiopathic autism: cellular and molecular phenotypes in pluripotent stem cell-derived neurons. Mol Neurobiol. 2017;54:4507-23.

87. DeRosa BA, El Hokayem J, Artimovich E, Garcia-Serje C, Phillips AW, Van Booven D, et al. Convergent pathways in idiopathic autism revealed by time course transcriptomic analysis of patient-derived neurons. Sci Rep. 2018;8:8423.

88. Marchetto MC, Belinson H, Tian Y, Freitas BC, Fu C, Vadodaria K, et al. Altered proliferation and networks in neural cells derived from idiopathic autistic individuals. Mol Psychiatry. 2017;22:820-35.

89. Schafer ST, Paquola ACM, Stern S, Gosselin D, Ku M, Pena M, et al. Pathological priming causes developmental gene network heterochronicity in autistic subject-derived neurons. Nat Neurosci. 2019;22:243-55.

90. Zeng L, Zhang P, Shi L, Yamamoto V, Lu W, Wang K. Functional impacts of NRXN1 knockdown on neurodevelopment in stem cell models. PLoS ONE. 2013;8:e59685.

91 Kim JJ, Savas JN, Miller MT, Hu X, Carromeu C, Lavallée-Adam M, et al. Proteomic analyses reveal misregulation of LIN28 expression and delayed timing of glial differentiation in human iPS cells with MECP2 loss-of-function. PLoS ONE. 2019;14:e0212553.

92. Williams EC, Zhong X, Mohamed A, Li R, Liu Y, Dong Q, et al. Mutant astrocytes differentiated from Rett syndrome patients-specific iPSCs have adverse effects on wild-type neurons. Hum Mol Genet. 2014;23:2968-80

93. Russo FB, Freitas BC, Pignatari GC, Fernandes IR, Sebat J, Muotri AR, et al. Modeling the interplay between neurons and astrocytes in autism using human induced pluripotent stem cells. Biol Psychiatry. 2018:83:569-78.

94. Merikangas AK, Calkins ME, Bilker WB, Moore TM, Gur RC, Gur RE. Parental age and offspring psychopathology in the Philadelphia neurodevelopmental cohort. J Am Acad Child Adolesc Psychiatry. 2017;56:391-400.

95. Durkin MS, Maenner MJ, Newschaffer CJ, Lee L-C, Cunniff CM, Daniels $J$, et al. Advanced parental age and the risk of autism spectrum disorder. Am J Epidemiol. 2008;168:1268-76.

96. Chess S. Autism in children with congenital rubella. J Autism Child Schizophr. 1971;1:33-47.

97. Atladottir HO, Henriksen TB, Schendel DE, Parner ET. Autism after infection, febrile episodes, and antibiotic use during pregnancy: an exploratory study. Pediatrics. 2012;130:e1447-e14541454.

98. Jiang H, Xu L, Shao L, Xia R, Yu Z, Ling Z, et al. Maternal infection during pregnancy and risk of autism spectrum disorders: a systematic review and meta-analysis. Brain Behav Immun. 2016;58:165-72. 
99 Baron-Cohen S, Tsompanidis A, Auyeung B, Nørgaard-Pedersen B, Hougaard DM, Abdallah M, et al. Foetal oestrogens and autism. Mol Psychiatry. 2020;25:2970-78.

100. Liew Z, Ritz B, Virk J, Olsen J. Maternal use of acetaminophen during pregnancy and risk of autism spectrum disorders in childhood: a Danish national birth cohort study. Autism Res. 2016;9:951-8.

101. Bauer AZ, Kriebel D. Prenatal and perinatal analgesic exposure and autism: an ecological link. Environ Health. 2013;12:41.

102. Avella-Garcia CB, Julvez J, Fortuny J, Rebordosa C, García-Esteban R, Galán IR, et al. Acetaminophen use in pregnancy and neurodevelopment: attention function and autism spectrum symptoms. Int J Epidemiol. 2016:45:1987-96.

103. Croen LA, Grether JK, Yoshida CK, Odouli R, Hendrick V. Antidepressant use during pregnancy and childhood autism spectrum disorders. Arch Gen Psychiatry. 2011;68:1104-12.

104. Christensen J, Grønborg TK, Sørensen MJ, Schendel D, Parner ET, Pedersen $\mathrm{LH}$, et al. Prenatal valproate exposure and risk of autism spectrum disorders and childhood autism. JAMA. 2013;309:1696-703.

105. Meador KJ, Baker GA, Browning N, Cohen MJ, Bromley RL, ClaytonSmith J, et al. Fetal antiepileptic drug exposure and cognitive outcomes at age 6 years (NEAD study): a prospective observational study. Lancet Neurol. 2013:12:244-52

106. Hofrichter M, Nimtz L, Tigges J, Kabiri Y, Schröter F, Royer-Pokora B, et al. Comparative performance analysis of human iPSC-derived and primary neural progenitor cells (NPC) grown as neurospheres in vitro. Stem Cell Res. 2017;25:72-82.

107. Miranda CC, Fernandes TG, Pinto SN, Prieto M, Diogo MM, Cabral JMS. A scale out approach towards neural induction of human induced pluripotent stem cells for neurodevelopmental toxicity studies. Toxicol Lett. 2018;294:51-60.

108. Chanda S, Ang CE, Lee QY, Ghebrial M, Haag D, Shibuya Y, et al. Direct reprogramming of human neurons identifies MARCKSL1 as a pathogenic mediator of valproic acid-induced teratogenicity. Cell Stem Cell. 2019;25(103-119):e6.

109. Volk HE, Lurmann F, Penfold B, Hertz-Picciotto I, McConnell R. Trafficrelated air pollution, particulate matter, and autism. JAMA Psychiatry. 2013;70:71.

110. Kalkbrenner AE, Windham GC, Serre ML, Akita Y, Wang X, Hoffman K, et al. Particulate matter exposure, prenatal and postnatal windows of susceptibility, and autism spectrum disorders. Epidemiology. 2015;26:30-42.

111. Raz R, Roberts AL, Lyall K, Hart JE, Just AC, Laden F, et al. Autism spectrum disorder and particulate matter air pollution before, during, and after pregnancy: a nested case-control analysis within the nurses health study II cohort. Environ Health Perspect. 2015;123:264-70.

112. Yamada S, Kubo Y, Yamazaki D, Sekino Y, Nomura Y, Yoshida S, et al. Tributyltin inhibits neural induction of human induced pluripotent stem cells. Sci Rep. 2018;8:12155.

113. Yamada S, Kubo Y, Yamazaki D, Sekino Y, Kanda Y. Chlorpyrifos inhibits neural induction via Mfn 1-mediated mitochondrial dysfunction in human induced pluripotent stem cells. Sci Rep. 2017:7:40925.

114. von Ehrenstein OS, Ling C, Cui X, Cockburn M, Park AS, Yu F, et al. Prenatal and infant exposure to ambient pesticides and autism spectrum disorder in children: population based case-control study. BMJ. 2019;364:1962

115. Carter CJ, Blizard RA. Autism genes are selectively targeted by environmental pollutants including pesticides, heavy metals, bisphenol A, phthalates and many others in food, cosmetics or household products. Neurochem Int. 2016;101:83-109.

116. Kern JK, Geier DA, Adams JB, Geier MR. A biomarker of mercury bodyburden correlated with diagnostic domain specific clinical symptoms of autism spectrum disorder. Biometals. 2010;23:1043-51.

117. Youn S-I, Jin S-H, Kim S-H, Lim S. Porphyrinuria in Korean children with autism: correlation with oxidative stress. J Toxicol Environ Heal Part A. 2010;73:701-10

118. Raciti M, Salma J, Spulber S, Gaudenzi G, Khalajzeyqami Z, Conti M, et al NRXN1 deletion and exposure to methylmercury increase astrocyte differentiation by different notch-dependent transcriptional mechanisms. Front Genet. 2019:10:593.
119. Pei Y, Peng J, Behl M, Sipes NS, Shockley KR, Rao MS, et al. Comparative neurotoxicity screening in human iPSC-derived neural stem cells, neurons and astrocytes. Brain Res. 2016;1638:57-73.

120. Kelava I, Lancaster MA. Stem cell models of human brain development. Cell Stem Cell. 2016;18:736-48.

121. Di Lullo E, Kriegstein AR. The use of brain organoids to investigate neural development and disease. Nat Rev Neurosci. 2017:18:573-84.

122 Benito-Kwiecinski S, Lancaster MA. Brain organoids: human neurodevelopment in a dish. Cold Spring Harb Perspect Biol. 2019;12:035709.

123. Wang Q, Charych El, Pulito VL, Lee JB, Graziane NM, Crozier RA, et al. The psychiatric disease risk factors DISC1 and TNIK interact to regulate synapse composition and function. Mol Psychiatry. 2011;16:1006-233.

124. Ye F, Kang E, Yu C, Qian X, Jacob F, Yu C, et al. DISC1 regulates neurogenesis via modulating kinetochore attachment of Ndel1/Nde1 during mitosis. Neuron. 2017;96:1041-1054.e5.

125. Srikanth P, Lagomarsino VN, Muratore CR, Ryu SC, He A, Taylor WM, et al. Shared effects of DISC1 disruption and elevated WNT signaling in human cerebral organoids. Transl Psychiatry. 2018;8:77.

126. Chaste P, Klei L, Sanders SJ, Murtha MT, Hus V, Lowe JK, et al. Adjusting head circumference for covariates in autism: clinical correlates of a highly heritable continuous trait. Biol Psychiatry. 2013;74:576-84.

127. Chawarska K, Campbell D, Chen L, Shic F, Klin A, Chang J. Early generalized overgrowth in boys with autism. Arch Gen Psychiatry. 2011:68:1021-31.

128. Lainhart JE, Bigler ED, Bocian M, Coon H, Dinh E, Dawson G, et al. Head circumference and height in autism: a study by the Collaborative Program of Excellence in Autism. Am J Med Genet A. 2006;140:2257-74.

129. Mariani J, Coppola G, Zhang P, Abyzov A, Provini L, Tomasini L, et al. FOXG1-dependent dysregulation of GABA/glutamate neuron differentiation in autism spectrum disorders. Cell. 2015;162:375-90.

130. Wang P, Mokhtari R, Pedrosa E, Kirschenbaum M, Bayrak C, Zheng D, et al. CRISPR/Cas9-mediated heterozygous knockout of the autism gene CHD8 and characterization of its transcriptional networks in cerebral organoids derived from iPS cells. Mol Autism. 2017;8:11.

131. Wang P, Lin M, Pedrosa E, Hrabovsky A, Zhang Z, Guo W, et al. CRISPR/ Cas9-mediated heterozygous knockout of the autism gene CHD8 and characterization of its transcriptional networks in neurodevelopment. Mol Autism. 2015;6:55.

132. Sugathan A, Biagioli M, Golzio C, Erdin S, Blumenthal I, Manavalan P, et al. CHD8 regulates neurodevelopmental pathways associated with autism spectrum disorder in neural progenitors. Proc Natl Acad Sci. 2014:111:E4468-E4477477.

133. Zhang W, Ma L, Yang M, Shao Q, Xu J, Lu Z, et al. Cerebral organoid and mouse models reveal a RAB39b-PI3K-mTOR pathway-dependent dysregulation of cortical development leading to macrocephaly/ autism phenotypes. Genes Dev. 2020;34:580-97.

134. Mellios N, Feldman DA, Sheridan SD, Ip JPK, Kwok S, Amoah SK, et al. MeCP2-regulated miRNAs control early human neurogenesis through differential effects on ERK and AKT signaling. Mol Psychiatry. 2018;23:1051-65

135. Birey F, Andersen J, Makinson CD, Islam S, Wei W, Huber N, et al. Assembly of functionally integrated human forebrain spheroids. Nature 2017:545:54-9.

136. Bagley JA, Reumann D, Bian S, Lévi-Strauss J, Knoblich JA. Fused cerebral organoids model interactions between brain regions. Nat Methods. 2017;14:743-51.

137. Xiang Y, Tanaka Y, Patterson B, Kang Y-J, Govindaiah G, Roselaar N, et al. Fusion of regionally specified hPSC-derived organoids models human brain development and interneuron migration. Cell Stem Cell. 2017:21:383-398.e7.

138. Bortone D, Polleux F. KCC2 expression promotes the termination of cortical interneuron migration in a voltage-sensitive calciumdependent manner. Neuron. 2009;62:53-71.

139 Real R, Peter M, Trabalza A, Khan S, Smith MA, Dopp J, et al. In vivo modeling of human neuron dynamics and Down syndrome. Science. 2018:362:eaau810.

140. Mansour AA, Gonçalves JT, Bloyd CW, Li H, Fernandes S, Quang D, et al. An in vivo model of functional and vascularized human brain organoids. Nat Biotechnol. 2018;36:432-41.

141. Xu R, Brawner AT, Li S, Liu J-J, Kim H, Xue H, et al. OLIG2 drives abnormal neurodevelopmental phenotypes in human ipsc-based organoid 
and chimeric mouse models of Down syndrome. Cell Stem Cell. 2019;24:908-926.e8.

142 Nassi JJ, Cepko CL, Born RT, Beier KT. Neuroanatomy goes viral! Front Neuroanat. 2015;9:80.

143. Byers B, Lee HJ, Liu J, Weitz AJ, Lin P, Zhang P, et al. Direct in vivo assessment of human stem cell graft-host neural circuits. Neuroimage. 2015;114:328-37.

144. Kumamaru H, Lu P, Rosenzweig ES, Kadoya K, Tuszynski MH. Regenerating corticospinal axons innervate phenotypically appropriate neurons within neural stem cell grafts. Cell Rep. 2019;26:2329-2339. e4.

145. Grealish S, Heuer A, Cardoso T, Kirkeby A, Jönsson M, Johansson J, et al. Monosynaptic tracing using modified rabies virus reveals early and extensive circuit integration of human embryonic stem cellderived neurons. Stem Cell Rep. 2015;4:975-83.

146. Doerr J, Schwarz MK, Wiedermann D, Leinhaas A, Jakobs A, Schloen F, et al. Whole-brain 3D mapping of human neural transplant innervation. Nat Commun. 2017;8:14162.

147. Hildebrand L, Seemann P, Kurtz A, Hecht J, Contzen J, Gossen M, et al. Selective cell targeting and lineage tracing of human induced pluripotent stem cells using recombinant avian retroviruses. Cell Mol Life Sci. 2015;72:4671-80.

148. Linaro D, Vermaercke B, Iwata R, Ramaswamy A, Libé-Philippot B, Boubakar $\mathrm{L}$, et al. Xenotransplanted human cortical neurons reveal species-specific development and functional integration into mouse visual circuits. Neuron. 2019;104:972-986.e6.

149. Brennand KJ, Simone A, Jou J, Gelboin-Burkhart C, Tran N, Sangar $S$, et al. Modelling schizophrenia using human induced pluripotent stem cells. Nature. 2011;473:221-5.

150. Hochbaum DR, Zhao Y, Farhi SL, Klapoetke N, Werley CA, Kapoor V, et al. All-optical electrophysiology in mammalian neurons using engineered microbial rhodopsins. Nat Methods. 2014;11:825-33.

151. Stroh A, Tsai H-C, Wang L-P, Zhang F, Kressel J, Aravanis A, et al. Tracking stem cell differentiation in the setting of automated optogenetic stimulation. Stem Cells. 2011;29:78-88.

152. Klapper SD, Sauter EJ, Swiersy A, Hyman MAE, Bamann C, Bamberg E, et al. On-demand optogenetic activation of human stem-cell-derived neurons. Sci Rep. 2017;7:14450.

153. Steinbeck JA, Choi SJ, Mrejeru A, Ganat Y, Deisseroth K, Sulzer D, et al. Optogenetics enables functional analysis of human embryonic stem cell-derived grafts in a Parkinson's disease model. Nat Biotechnol. 2015:33:204-9.

154. Qi Y, Zhang X-J, Renier N, Wu Z, Atkin T, Sun Z, et al. Combined small-molecule inhibition accelerates the derivation of functional cortical neurons from human pluripotent stem cells. Nat Biotechnol. 2017;35:154-63.

155. Bürgers J, Pavlova I, Rodriguez-Gatica JE, Henneberger C, Oeller M, Ruland JA, et al. Light-sheet fluorescence expansion microscopy: fast mapping of neural circuits at super resolution. Neurophotonics. 2019;6:015005.

156. Plumbly W, Brandon N, Deeb TZ, Hall J, Harwood AJ. L-type voltagegated calcium channel regulation of in vitro human cortical neuronal networks. Sci Rep. 2019:9:13810.

157. Trujillo CA, Gao R, Negraes PD, Gu J, Buchanan J, Preissl S, et al. Complex oscillatory waves emerging from cortical organoids model early human brain network development. Cell Stem Cell. 2019;25:558-569.e7.

158. Livet J, Weissman TA, Kang H, Draft RW, Lu J, Bennis RA, et al. Transgenic strategies for combinatorial expression of fluorescent proteins in the nervous system. Nature. 2007:450:56-62.

159 Sakaguchi R, Leiwe MN, Imai T. Bright multicolor labeling of neuronal circuits with fluorescent proteins and chemical tags. eLife. 2018;7:e40350.

160. Schwarz LA, Miyamichi K, Gao XJ, Beier KT, Weissbourd B, DeLoach KE, et al. Viral-genetic tracing of the input-output organization of a central noradrenaline circuit. Nature. 2015;524:88-92.

161. Kim J, Zhao T, Petralia RS, Yu Y, Peng H, Myers E, et al. mGRASP enables mapping mammalian synaptic connectivity with light microscopy. Nat Methods. 2011;9:96-102.

162. Song JH, Lucaci D, Calangiu I, Brown MTC, Park JS, Kim J, et al. Combining mGRASP and optogenetics enables high-resolution functional mapping of descending cortical projections. Cell Rep. 2018;24:1071-80.
163. Kebschull JM, Garcia da Silva P, Reid AP, Peikon ID, Albeanu DF, Zador AM. High-throughput mapping of single-neuron projections by sequencing of barcoded RNA. Neuron. 2016;91:975-87.

164. Peikon ID, Kebschull JM, Vagin W, Ravens DI, Sun Y-C, Brouzes E, et al. Using high-throughput barcode sequencing to efficiently map connectomes. Nucleic Acids Res. 2017;45:e115.

165. Chen X, Sun Y-C, Zhan H, Kebschull JM, Fischer S, Matho K, et al. Highthroughput mapping of long-range neuronal projection using in situ sequencing. Cell. 2019;179:772-786.e19.

166. Fosque BF, Sun Y, Dana H, Yang C-T, Ohyama T, Tadross MR, et al. Neural circuits labeling of active neural circuits in vivo with designed calcium integrators. Science. 2015;347:755-60.

167. Lee D, Hyun JH, Jung K, Hannan P, Kwon H-B. A calcium- and lightgated switch to induce gene expression in activated neurons. Nat Biotechnol. 2017;35:858-63.

168. Wang W, Wildes CP, Pattarabanjird T, Sanchez MI, Glober GF, Matthews $\mathrm{GA}$, et al. A light- and calcium-gated transcription factor for imaging and manipulating activated neurons. Nat Biotechnol. 2017;35:864-71.

169. Airan RD, Thompson KR, Fenno LE, Bernstein H, Deisseroth K. Temporally precise in vivo control of intracellular signalling. Nature. 2009;458:1025-9.

170. Siuda ER, Copits BA, Schmidt MJ, Baird MA, Al-Hasani R, Planer WJ, et al. Spatiotemporal control of opioid signaling and behavior. Neuron. 2015:86:923-35.

171. Li Y, He Y, Chen M, Pu Z, Chen L, Li P, et al. Optogenetic activation of adenosine A2A receptor signaling in the dorsomedial striatopallidal neurons suppresses goal-directed behavior. Neuropsychopharmacology. 2016;41:1003-13.

172. Zheng W, Zhou J, Luan Y, Yang J, Ge Y, Wang M, et al. Spatiotemporal control of GPR37 signaling and its behavioral effects by optogenetics. Front Mol Neurosci. 2018:11:95.

173. Marvin JS, Borghuis BG, Tian L, Cichon J, Harnett MT, Akerboom J, et al. An optimized fluorescent probe for visualizing glutamate neurotransmission. Nat Methods. 2013;10:162-70.

174. Nguyen Q-T, Schroeder LF, Mank M, Muller A, Taylor P, Griesbeck O, et al. An in vivo biosensor for neurotransmitter release and in situ receptor activity. Nat Neurosci. 2010;13:127-32.

175. Marvin JS, Shimoda Y, Magloire V, Leite M, Kawashima T, Jensen TP, et al. A genetically encoded fluorescent sensor for in vivo imaging of GABA. Nat Methods. 2019;16:763-70.

176. Sun F, Zeng J, Jing M, Zhou J, Feng J, Owen SF, et al. A genetically encoded fluorescent sensor enables rapid and specific detection of dopamine in flies, fish, and mice. Cell. 2018;174:481-496.e19.

177. Feng J, Zhang C, Lischinsky JE, Jing M, Zhou J, Wang H, et al. A genetically encoded fluorescent sensor for rapid and specific in vivo detection of norepinephrine. Neuron. 2019;102:745-761.e8.

178. Kumari D, Swaroop M, Southall N, Huang W, Zheng W, Usdin K. Highthroughput screening to identify compounds that increase fragile $X$ mental retardation protein expression in neural stem cells differentiated from fragile $X$ syndrome patient-derived induced pluripotent stem cells. Stem Cells Transl Med. 2015;4:800-8.

179. Kaufmann M, Schuffenhauer A, Fruh I, Klein J, Thiemeyer A, Rigo P, et al. High-throughput screening using iPSC-derived neuronal progenitors to identify compounds counteracting epigenetic gene silencing in fragile X syndrome. J Biomol Screen. 2015;20:1101-11.

180. Vershkov D, Fainstein N, Suissa S, Golan-Lev T, Ben-Hur T, Benvenisty N. FMR1 reactivating treatments in fragile X iPSC-derived neural progenitors in vitro and in vivo. Cell Rep. 2019;26:2531-2539.e4.

181. Darville H, Poulet A, Rodet-Amsellem F, Chatrousse L, Pernelle J, Boissart C, et al. Human pluripotent stem cell-derived cortical neurons for high throughput medication screening in autism: a proof of concept study in SHANK3 haploinsufficiency syndrome. EBioMedicine. 2016;9:293-305.

182. Mancuso R, Van Den Daele J, Fattorelli N, Wolfs L, Balusu S, Burton O, et al. Stem-cell-derived human microglia transplanted in mouse brain to study human disease. Nat Neurosci. 2019;22:2111-6.

\section{Publisher's Note}

Springer Nature remains neutral with regard to jurisdictional claims in published maps and institutional affiliations. 